\title{
Lessons Learned from Aquifer Storage and Recovery (ASR) Systems in the United States
}

\author{
Frederick Bloetscher ${ }^{1}$, Chi Ho Sham ${ }^{2}$, Joseph J. Danko III ${ }^{3}$, Samuel Ratick ${ }^{4}$ \\ ${ }^{1}$ Florida Atlantic University, Boca Raton, USA \\ ${ }^{2}$ The Cadmus Group, Inc., Waltham, USA \\ ${ }^{3}$ University of Connecticut, Storr, USA \\ ${ }^{4}$ Clark University, Worcester, USA \\ Email: h2o man@bellsouth.net
}

Received 15 September 2014; revised 12 October 2014; accepted 5 November 2014

Copyright (C) 2014 by authors and Scientific Research Publishing Inc.

This work is licensed under the Creative Commons Attribution International License (CC BY). http://creativecommons.org/licenses/by/4.0/

cC) (7) Open Access

\begin{abstract}
This paper is the result of a survey and analyses of available data from 204 Aquifer Storage and Recovery (ASR) sites in the United States. This ASR site survey included all active and inactive sites and collected both operational and construction details. The inactive sites are of particular interest here because these are the projects from which valuable lessons can often be learned. The intent of this paper is to examine the reasons for those projects that are terminated. Statistical analyses indicated that there were factors associated with terminated ASR projects: general geographic location (e.g., region), operational issue, storage cycle, casing material, and injection formation. The injection formation involves local geology and aquifer characteristics (i.e., whether the aquifer is leaky and/or unconfined, and if water can be displaced to surface water bodies or adjacent aquifers). Operational problems associated with inactive projects include well clogging, metals mobilization, a low percentage of recovery for injected water, and disinfection byproducts in the recovered water.
\end{abstract}

\section{Keywords}

Aquifer Storage and Recovery, Inactive, Clogging, Recovery

\section{Introduction}

In some areas of the world, treated or treatable water is being injected and stored beneath the earth's surface in aquifers (i.e., aquifer storage programs) to preserve current water resources for future uses, plan for future droughts, retain instead of releasing surplus water during times of plenty, recharge wellfields and/or store water 
for use at a later point in time to sustain development [1]. As a result, most aquifer storage programs are found in arid areas (with consistent water deficits) or coastal areas (subjected to salt water intrusion) or areas with pronounced seasonal wet and dry periods (mismatched demands and supplies). The beneficial use of water that is not needed at the present is the main driver for the various aquifer storage programs. To date, all aquifer storage strategies are proven, comparatively cost-effective programs that are capable of successfully managing existing water resources to increase reliability for long-term water supply. But that does not mean all such programs are or will be successful to the client.

For the purpose of this discussion, AWWA Manual M21 on Groundwater [1] divides aquifer storage programs into four categories: Artificial Aquifer Creation, Aquifer Recharge, Aquifer Reclamation, and Aquifer Storage and Recovery (ASR). All of these approaches are used by the water supply industry to insure that reliable water resources are available for agricultural, environmental and urban uses. The focus of this paper is on ASR projects which are different from the other aquifer storage strategies because the associated injection and recovery wells are generally the same wells.

ASR has been a viable approach in the management of both potable and non-potable water supplies. In general, ASR technology can be used to increase the efficiency of water system operations because during the wet, low-demand portion of the year, some or all of the unused water treatment plant capacity can be used to treat water and store it in an aquifer for later retrieval and usage. Common applications are the injection of potable or raw water into an aquifer, with the intention to provide future withdrawal for augmentation of water supplies at a later time. The storage period can be over multiple months to allow the stored water to meet the next high demand season or an emergency (e.g., a severe drought or an extreme flood) or during an interruption of water withdrawal (e.g., due to equipment breakdown or water right dispute). Figure 1 shows the concept behind ASR graphically [2].

A benefit of ASR technology in areas with significant fluctuations in raw water supplies and/or demands during the year is that it may allow the water utility to size its treatment plants for average conditions rather than seasonal high demands; thereby saving capital infrastructure costs. Once treated, potable water is often stored and recovered to deal with unmet demands. The use of potable water in ASR projects often allow the water utility to withdraw the injected water, disinfect it, and pump it directly to the distribution system without much further treatment, although this objective may not be met for various reasons. Other sources may require treatment after recovery like untreated raw water surface and ground sources, stormwater runoff and reclaimed water (Englewood, FL, Las Vegas Valley and several Arizona wells do this among others). All have been captured, injected, stored, and recovered.

In the United States, ASR wells are covered under U.S. Environmental Protection Agency (EPA)'s Underground Injection Control (UIC) program that was promulgated under the Safe Drinking Water Act (SDWA) Sections 1421 through 1426. U.S. EPA's authority to govern underground injection programs is codified at 40 Code of Federal Regulation (CFR) 144 through 148. The purpose of the rules is to protect underground sources of drinking water (USDWs ${ }^{1}$ ). The UIC program requirements were developed to ensure that emplacement of fluids via injection wells did not endanger current and future USDWs. Much of the UIC regulations were aimed at regulating disposal of waste fluids via underground injection, and focused on the injection of hazardous wastes (Bloetscher, et al., 2002). As a part of delegation of federal programs to the states, 40 states and territories, along with 2 tribes, now administer all or a portion of the UIC programs in their jurisdiction [1]. Under the auspices of protecting public health, the regulatory agencies have the authority to require, accept, and reject permits for underground injection wells on the basis of their successes or failures to protect USDWs.

Based on a survey conducted in 2013, there are 204 ASR sites in the United States [3]. Data were collected for each site via the survey and subsequent follow-up phones and email communication. As a part of this survey, the status of the various ASR systems was obtained and reviewed. Valuable information can be drawn from the data and analyses regarding lessons learned on the various ASR projects. Figure 2 shows the status of the ASR wells by site. Approximately $37 \%$ of the ASR sites are considered to be operational, while $24 \%$ are under various stages of testing. In addition, feasibility studies were conducted at $12 \%$ of the surveyed sites but these projects were not pursued further (as of the date of the survey); while $26 \%$ of the sites were associated with the cessation of ASR activities (see Figure 2). It is these inactive projects that are the focus of this paper and their

${ }^{1}$ An USDW as defined at 40 CFR 144.3 is an aquifer or its portion: 1) a) which supplies any public water system; or b) which contains a sufficient quantity of ground water to supply a public water system; and i) currently supplies drinking water for human consumption; or ii) contains fewer than $10,000 \mathrm{mg} / \mathrm{l}$ total dissolved solids; and 2) which is not an exempted aquifer. 


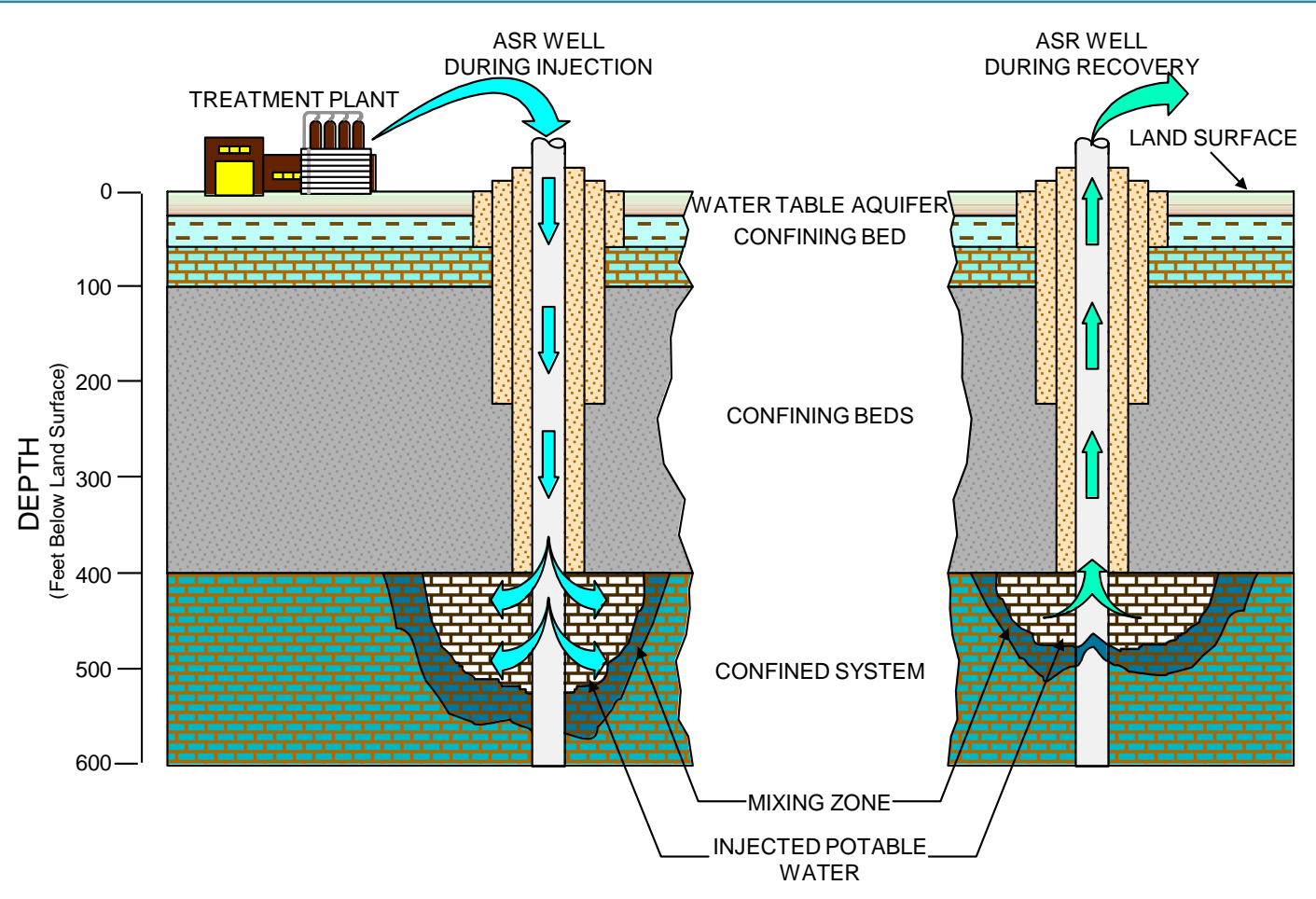

Figure 1. Aquifer storage and recovery concept.

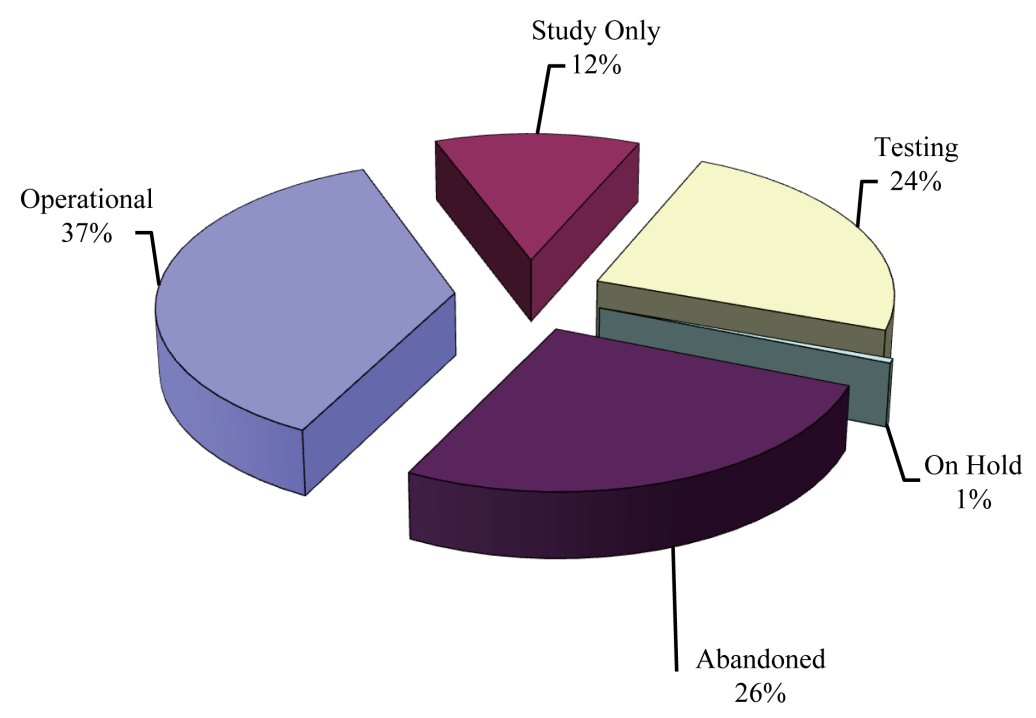

Figure 2. Status of ASR project sites.

failures would help provide insight into the challenges associated with the ASR technology. For instance, inactive and discontinued project sites comprise $26 \%$ of the 204 sites, and a total of 220 wells (see Table 1), indicating that ASR projects are not always successful. While a lot can be learned from successful projects, reviewing the failed systems provides insights into some of the criteria and process associated with the development of ASR programs.

\section{Methodology}

The project comprised two phases, data collection and data analysis. Data were collected in two steps. States and U.S. EPA were contacted by phone or email to track down the regulations that govern ASR programs from state 
Table 1. Summary of inactive ASR programs.

\begin{tabular}{|c|c|c|c|c|c|}
\hline State & Project/Location & $\begin{array}{l}\text { Number of Wells } \\
\text { in the Project }\end{array}$ & $\begin{array}{l}\text { Number of } \\
\text { Abandoned Wells } \\
\text { in the Project }\end{array}$ & Source Water & $\begin{array}{l}\text { Reason For } \\
\text { Abandon }\end{array}$ \\
\hline $\mathrm{AZ}$ & Chandler Intel & 4 & 3 & River Water & Clogging \\
\hline $\mathrm{AZ}$ & Gilbert & 2 & 1 & Reclaimed RO & Clogging \\
\hline $\mathrm{AZ}$ & North Scottsdale & 3 & 2 & Raw Surface & Clogging \\
\hline $\mathrm{AZ}$ & Peoria-Stone Street & 1 & 1 & Raw Surface & Clogging \\
\hline $\mathrm{AZ}$ & Phoenix 1-Salt River Project & 8 & 8 & Raw Surface & Clogging \\
\hline $\mathrm{AZ}$ & Phoenix 2-Tormanto & 2 & 2 & Raw Surface & Clogging \\
\hline $\mathrm{AZ}$ & Phoenix 3-Cave Creek & 2 & 2 & Recalimed & Clogging \\
\hline CA & Antelope Valley Los Angeles PWD & 2 & 2 & Ground & \\
\hline CA & $\begin{array}{l}\text { Boron CSD well } 1558 \text { \& gephardt } \\
\text { water fr AV ab project no water } \\
\text { provided-shortage retro fit } 1967 \text { well }\end{array}$ & 1 & 1 & Surface & No Water \\
\hline CA & Camarillo & 2 & 2 & Surface & Recovery \\
\hline CA & East Bay MUD & 1 & 1 & Surface & \\
\hline CA & $\begin{array}{c}\text { Eastern Municipal Water District, } \\
\text { Perris (follic) }\end{array}$ & 1 & 1 & Surface & Mn, Thm \\
\hline CA & Fresno & 1 & 1 & Surface & Unknown \\
\hline CA & Oxnard, City of & 5 & 5 & Surface & Clogging \\
\hline CA & PALo Alto & 1 & 1 & Surface & Unknown \\
\hline CA & Pasadena, City of (thm) & 3 & 3 & Surface & Thm \\
\hline CA & Pleasanton, Zone 7 Livermore & 4 & 4 & Surface & \\
\hline CA & $\begin{array}{l}\text { Santa Clara County Water } \\
\text { District, San Jose }\end{array}$ & 1 & 1 & Surface & \\
\hline CA & $\begin{array}{l}\text { Sonoma County Water Agency-Santa } \\
\text { Rosa ASR } 1 \text { aband to std wll }\end{array}$ & 1 & 1 & Surface & Conv To Well \\
\hline CA & Sweetwater/National City & 2 & 2 & Surface & Clogging \\
\hline CA & Tracy, City of & 2 & 1 & Surface & Clogging \\
\hline CA & Valley Water Company Foothills & 2 & 2 & Surface & Clogging \\
\hline CA & Valley Water District Monk Hill & 4 & & Surface & \\
\hline CA & Victorville (Victor Valley Water District) & 4 & 4 & Surface & \\
\hline $\mathrm{CO}$ & Castle Pines North (Worley) AR4 & 2 & 2 & Surface & Recovery \\
\hline $\mathrm{CO}$ & $\begin{array}{l}\text { Colorado Springs Utilitie } \\
\text { Park Forest/Donata }\end{array}$ & 2 & 2 & Surface & Recovery \\
\hline $\mathrm{CO}$ & $\begin{array}{c}\text { East Cherry Creek Valley Water } \\
\text { District and Sanitation/Willows } \\
\text { Water District acq } 2001\end{array}$ & 4 & 4 & Surface & Recovery \\
\hline $\mathrm{CO}$ & Parker Water and Sanitation District & 1 & 1 & Surface & Recovery \\
\hline FL & Bonita Springs San Carlos & 1 & 1 & Drinking Water & Arsenic \\
\hline FL & Bradenton-Reservoir As & 1 & 1 & Surface & Arsenic \\
\hline
\end{tabular}




\section{Continued}

\begin{tabular}{|c|c|c|c|c|c|}
\hline FL & Broward County & 1 & 1 & Raw GW & Recovery \\
\hline FL & CERP Hillsboro Basin & 1 & 1 & Surface & Arsenic \\
\hline FL & CERP Lake Okeechobee & 1 & 1 & Surface Water & Arsenic \\
\hline FL & CERP St Lucie & 1 & 1 & Raw GW & Arsenic \\
\hline FL & CERP Taylor Creek SFWMD (Cl 61k) & 1 & 1 & Surface & Arsenic \\
\hline FL & Collier County North & 1 & 1 & Reclaimed & Arsenic \\
\hline FL & Collier County, Manatee Rd & 1 & 1 & Drinking Water & Permit Expired \\
\hline FL & Deerfield Beach, City of & 1 & 1 & Ground & Recovery \\
\hline FL & Delray Beach & 1 & 1 & PWS GW & Recovery \\
\hline FL & Englewood & 1 & 1 & Reclaimed & Arsenic \\
\hline FL & Fort Lauderdale Fiveash & 1 & 1 & Raw GW & Recovery \\
\hline FL & Ft. Myers Winkler & 1 & 1 & Drinking Water & Clogging \\
\hline FL & Hialeah & 1 & 1 & Raw GW & Unknown \\
\hline FL & Lee County, Corkscrew & 6 & 6 & Drinking Water & Recovery \\
\hline FL & Lee County, North Reservoir & 1 & 1 & Surface & Clogging \\
\hline FL & Lee County, Olga WTP (as) & 1 & 1 & Ground & Arsenic \\
\hline FL & Manatee Southwest & 1 & 1 & Reclaimed & Unknown \\
\hline FL & Marathon & 1 & 1 & Drinking Water & Recovery \\
\hline FL & Miami Beach & 1 & 1 & Drinking Water & Recovery \\
\hline FL & Miami-Dade Water \& Sewer Dept. NW & 3 & 3 & Raw GW & Recovery \\
\hline FL & North Port s & 1 & 1 & Reclaimed & Unknown \\
\hline FL & NW Hillsborough (WQ deter) & 1 & 1 & Reclaimed & WQ Deterioration \\
\hline FL & PBC 3 & 1 & 1 & Raw GW & Recovery \\
\hline FL & PBC System 9 Hillsboro & 1 & 1 & Surface & Recovery \\
\hline FL & Punta Gorda-hell Creek & 4 & 4 & Drinking Water & Arsenic \\
\hline FL & SFWMD Jupiter & 1 & 1 & Raw GW & Recovery \\
\hline FL & St. Lucie Co SFWMD & 1 & 1 & Surface & Unknown \\
\hline FL & St. Petersburg, City of & 2 & 2 & Reclaimed & Unknown \\
\hline FL & Sunrise Springtree & 1 & 1 & Drinking Water & Recovery \\
\hline FL & West Palm Beach & 1 & 1 & Surface & Recovery \\
\hline GA & $\begin{array}{l}\text { Dalton (carpet industry-small test } \\
\text { wells only-no formation) }\end{array}$ & 5 & 5 & Raw GW & Recovery \\
\hline ID & Boise, United Water & 5 & 1 & & Clogging \\
\hline NJ & Monroe Township Municipal Util Auth & 2 & 2 & Ground & Unknown \\
\hline NM & Alamogordo & 1 & 1 & Ground & \\
\hline NV & N Las Vegas & 10 & 6 & Varies, Icn Reuse & Clogging \\
\hline NV & Washoe County (Truckee) gravity flow & 4 & 2 & Surface & Clogging \\
\hline
\end{tabular}




\begin{tabular}{|c|c|c|c|c|c|}
\hline \multicolumn{6}{|c|}{ Continued } \\
\hline NY & Bay Park (clog) & 1 & 1 & Pws & Clogging \\
\hline NY & East Meado Reclaimed Water (Clog) & 5 & 5 & Reclaimed & Clogging \\
\hline OR & Clackamas River Water (radon) & 1 & 1 & Surface & Radon \\
\hline OR & Pendleton, City of & 2 & 2 & Surface Via RBF & Clogging \\
\hline OR & Portland Water Bureau (Shore) & 7 & 2 & Surface & \\
\hline OR & Tigard, City of & 2 & 1 & Surface & \\
\hline OR & Tualatin, City of & 1 & 1 & Pws & \\
\hline SC & $\begin{array}{l}\text { Mt Pleasant Waterworks \& } \\
\text { Sewer Commission }\end{array}$ & 3 & 1 & Surface Water & Unknown \\
\hline SC & Myrtle Beach & 1 & 1 & Surface Water & WQ Deterioration \\
\hline SD & Huron (used prod wells) & 6 & 6 & Surface Water & \\
\hline $\mathrm{TN}$ & Memphis Light Gas and Water & 1 & 1 & Ground & \\
\hline $\mathrm{TX}$ & Laredo & 4 & 4 & Surface & Recovery \\
\hline UT & $\begin{array}{l}\text { Cachw/Leamington Town } \\
\text { Culinary Water Well }\end{array}$ & 1 & 1 & Ground & Clogging \\
\hline UT & $\begin{array}{l}\text { Jordan Valley Water District } \\
\text { (retro wells, clogging) }\end{array}$ & 18 & 18 & Surface & Clogging \\
\hline UT & Salt Lake City/High Plains? Clog & 40 & 40 & Surface & Clogging \\
\hline VA & Norfolk & 1 & 1 & Surface & Test Only \\
\hline WA & Lakehaven/Tacoma & 2 & 2 & Surface & Unknown \\
\hline WA & Sammamish Water & 3 & 3 & Surface & Unknown \\
\hline WA & Seattle Public Utilities Highline radon & 3 & 3 & Surface & Radon \\
\hline WA & Walla Walla & 2 & 2 & Surface & Unknown \\
\hline WA & Yakima & 1 & 1 & Pws & THM \\
\hline WI & Green Bay Utlility & 1 & 1 & Surface & Metals Mobilization \\
\hline \multirow[t]{2}{*}{ WI } & Oak Creek & 2 & 2 & Surface & Metals Mobilization \\
\hline & TOTAL & 246 & 220 & & \\
\hline
\end{tabular}

regulatory agencies and to determine whether the state had such programs in place or not. Note that there is a lack of ASR specific regulations at the federal level because the states are only required to submit inventory information on Class V wells and ensure non-endangerment of USDWs. While prior inventories were prepared by regulatory agencies and consultants, none were complete, comprehensive, and inclusive of all inactive projects. The regulatory investigations undertaken for this paper yielded a comprehensive list of ASR sites in the U.S., which was a critical component of the project. For the ASR projects included in the survey, variables of interest were used to account for operations, physical construction, and localized differences. These variables were extracted from the data sets. During the data extraction process, a number of wells were found to be older and/or not in service. In addition, data for some ASR sites were lost or missing. A confounding problem is that some states (e.g., California) have no centralized data sources, so there is a lack of consistency in the parameters collected.

The dataset collected for this project is the latest and most comprehensive representation of the ASR site inventory as of July 1, 2013. Data collected included:

- State (where the ASR site is located);

- Date the program was initiated or first well drilled; 
- Stage of development/status, categorized as study, testing, operational or abandoned;

- Number of wells drilled: the number of individual ASR wells onsite to accommodate the designed injection capacity;

- Number of abandoned wells, the number of individual ASR wells onsite no longer in service;

- Source of water, categorized as ground, surface, reclaimed of industrial water;

- Use of recovered water, categorized by irrigation, potable water supplies, raw water supplies, and surface water augmentation;

- Number of storage cycles (estimated), which is indicative of age of the system;

- Injection rate per well as a measure of injection capacity (converted to millions of gallons per day (MGD));

- Withdrawal rate per well as a measure of withdrawal capacity (converted to MGD);

- Peak flow as a measure of total available capacity on the site (converted to MGD);

- Total water stored (converted to millions of gallons);

- Depth of well casing below the surface: A measure of depth in feet of the most interior and deepest well casing that is installed at the final construction stage;

- Depth of well below the surface: A measure of depth in feet of the deepest point of the well;

- Casing material which is the final casing categorized as steel, PVC, fiberglass or stainless steel, separating stainless steel screens form stainless steel casings;

- Casing diameter which is a measure of diameter in inches of the most interior and deepest well casing that is installed at the final construction stage;

- Whether tubing and packer wells were used;

- Injection zone formation category, categorized into formation type such as limestone, sand, sandstone, basalt, and alluvial formation;

- Injection zone transmissivity or hydraulic conductivity (converted to transmissivity); as the measure the ability of water to maneuver through a porous media (the rate of flow per unit time per unit cross-sectional area, which is converted to gallons per day/ft);

- Total dissolved solids (TDS) of the water in the injection formation fluid;

- Type of confinement (formation type) categorized into formation type such as clay, dolomite, silt, shale, sandstone, basalt, or no confinement;

- Number of monitoring wells if known; and

- Operational issues reported. This is the area that provides much information on the inactive wells.

It should be noted that not all of the data listed above were available for all wells in the survey, especially the aquifer parameters associated with the older wells (e.g., TDS and hydraulic conductivity).

\section{Methods of Data Analysis}

This study examines a set of 24 ASR data elements, including 15 continuous and 9 categorical variables. Continuous variables are estimated start date, number of active wells, number of abandoned wells, injection capacity, withdrawal capacity, in/out ratio, peak flow on site, amount of water stored, depth of well, depth of casing, injection horizon, diameter of casing, transmissivity, total dissolved solids (TDS), and number of monitoring wells. Descriptive statistics for the 15 continuous variables are summarized in Table 2(a). Categorical variables are geographic region, issues arisen for an ASR program, water source, water use, number of storage cycles (there were grouped because of the range of numbers), casing material, tubing and packer (T\&P) code, injection formation code and confinement unit formation code. Descriptive statistics for the 9 categorical variables are summarized in Table 2(b). Geographic regions are summarized in Table 3. Table 4 shows the summary of logistic regression statistics predicting ASR success in the United States by region. Statistically significant coefficients are highlighted in bold and include issues or the lack thereof predominate by region with clogging, metals migration and recovery as the major issues (95\% confidence).

A Chi-square test was performed to determine if there is a statistically difference between program status (i.e., active versus inactive) and each of the categorical variables (Table 5). A Chi-square test is a statistical test to determine if there is a statistically relationship between two categorical variables. When the association between two categorical variables is statistically significant, a contingency table of the residual values would reveal if these values make sense based on a priori knowledge of the two phenomena being compared (Table 5). Residual values are calculated by subtracting the observed value $\left(O_{i j}\right)$ by the expected value $\left(E_{i j}\right)$ in each cell of a 
Table 2. (a) Descriptive statistics of continuous variables related to the ASR sites in the United States; (b) Descriptive statistics of categorical variables per ASR program status in the United States.

(a)

\begin{tabular}{|c|c|c|c|c|c|c|}
\hline & Observation & Missing & Minimum & Maximum & Average & Standard Deviation \\
\hline Estimated Start Date & 204 & 0 & 1963 & 2014 & 1999 & 10 \\
\hline Active & 75 & 0 & 1968 & 2010 & 1996 & 8 \\
\hline Inactive & 55 & 0 & 1963 & 2007 & 1995 & 10 \\
\hline Test/Study & 74 & 0 & 1970 & 2014 & 2004 & 9 \\
\hline Number of Active Wells & 201 & 3 & 0 & 87 & 4 & 8 \\
\hline Active & 75 & 0 & 0 & 87 & 6 & 11 \\
\hline Inactive & 54 & 1 & 0 & 40 & 3 & 5 \\
\hline Test/Study & 72 & 2 & 0 & 15 & 2 & 2 \\
\hline Number of Inactive Wells & 201 & 3 & 0 & 40 & 1 & 3 \\
\hline Active & 75 & 0 & 0 & 18 & 1 & 2 \\
\hline Inactive & 54 & 1 & 0 & 40 & 3 & 5 \\
\hline Test/Study & 72 & 2 & 0 & 5 & 0 & 1 \\
\hline Number of Monitoring Wells & 203 & 1 & 0 & 10 & 1 & 1 \\
\hline Active & 74 & 1 & 0 & 6 & 1 & 1 \\
\hline Inactive & 55 & 0 & 0 & 10 & 1 & 2 \\
\hline Test/Study & 74 & 0 & 0 & 7 & 0 & 1 \\
\hline Injection Capacity & 178 & 26 & 0 & 15 & 1.4 & 1.9 \\
\hline Active & 73 & 2 & 0.1 & 10 & 1.3 & 1.6 \\
\hline Inactive & 49 & 6 & 0 & 9 & 1.4 & 1.7 \\
\hline Test/Study & 56 & 18 & 0 & 15 & 1.5 & 2.4 \\
\hline Withdrawal Capacity & 180 & 24 & 0 & 15 & 1.9 & 2.2 \\
\hline Active & 74 & 1 & 0.1 & 10 & 1.9 & 1.9 \\
\hline Inactive & 52 & 3 & 0 & 9 & 1.9 & 2 \\
\hline Test/Study & 54 & 20 & 0 & 15 & 2.1 & 2.7 \\
\hline In/Out Ratio & 174 & 30 & 0.02 & 5.25 & 0.9 & 0.5 \\
\hline Active & 73 & 2 & 0.06 & 5.25 & 0.9 & 0.7 \\
\hline Inactive & 48 & 7 & 0.19 & 1.02 & 0.8 & 0.3 \\
\hline Test/Study & 53 & 21 & 0.02 & 2.5 & 0.8 & 0.4 \\
\hline Peak Flow on Site (MGD) & 175 & 29 & 0 & 40 & 3.9 & 5.3 \\
\hline Active & 73 & 2 & 0.1 & 23 & 4.7 & 4.9 \\
\hline Inactive & 49 & 9 & 0 & 23.7 & 3.3 & 4 \\
\hline Test/Study & 53 & 21 & 0 & 40 & 3.6 & 6.7 \\
\hline
\end{tabular}




\section{Continued}

\begin{tabular}{|c|c|c|c|c|c|c|}
\hline Amount of Water Stored (MG) & 162 & 42 & 0 & 78,400 & 1282.1 & 6262.5 \\
\hline Active & 69 & 6 & 0.2 & 78,400 & 2166.5 & 9462 \\
\hline Inactive & 46 & 9 & 0 & 3800 & 654 & 1039.3 \\
\hline Test/Study & 47 & 27 & 0 & 8400 & 598.4 & 1347.5 \\
\hline Depth of Well (ft) & 181 & 23 & 33 & 3882 & 801.3 & 560.5 \\
\hline Active & 73 & 2 & 75 & 2523 & 789.7 & 489.7 \\
\hline Inactive & 52 & 3 & 33 & 1770 & 728.3 & 456.1 \\
\hline Test/Study & 56 & 18 & 50 & 3882 & 884.2 & 713.6 \\
\hline Depth of Casing (ft) & 178 & 26 & 9 & 3832 & 594.6 & 489.4 \\
\hline Active & 50 & 5 & 39 & 2185 & 550.5 & 424.8 \\
\hline Inactive & 52 & 3 & 10 & 1457 & 561.4 & 371.9 \\
\hline Test/Study & 56 & 18 & 9 & 3832 & 680.5 & 636.4 \\
\hline Injection Horizon & 159 & 45 & 7.5 & 1501 & 225.6 & 234.3 \\
\hline Active & 65 & 10 & 7.5 & 1000 & 231 & 214.8 \\
\hline Inactive & 46 & 9 & 12 & 1186 & 200.2 & 228 \\
\hline Test/Study & 48 & 26 & 21 & 1501 & 242.7 & 266.3 \\
\hline Diameter of Casing (ft) & 136 & 68 & 6 & 40 & 14.9 & 5.3 \\
\hline Active & 56 & 19 & 6 & 26 & 14.5 & 4.2 \\
\hline Inactive & 43 & 12 & 6 & 40 & 15.6 & 6.9 \\
\hline Test/Study & 37 & 37 & 6 & 24 & 14.7 & 4.7 \\
\hline Transmissivity (gpd/sf) & 127 & 77 & 0.1 & 620,136 & 75534.6 & 137647.4 \\
\hline Active & 30 & 45 & 1 & 620,136 & 79157.60 & 154511.30 \\
\hline Inactive & 21 & 34 & 1.2 & 264,000 & 56732.30 & 90237.60 \\
\hline Test/Study & 26 & 48 & 0.1 & 600,000 & 86540.8 & 151498.6 \\
\hline Total Dissolved Solids (TDS) (ppm) & 66 & 138 & 50 & 6000 & 1563.50 & 1732.50 \\
\hline Active & 23 & 52 & 150 & 5500 & 1117.10 & 1569.50 \\
\hline Inactive & 28 & 27 & 140 & 6000 & 1896.40 & 1783.40 \\
\hline Test/Study & 15 & 59 & 50 & 6000 & 1631 & 1837.50 \\
\hline
\end{tabular}

(b)

\begin{tabular}{ccccccccc}
\hline & Observations & Missing & Basin Range & California & Mid-Atlantic & Mid-West & Pacific NW & SE Coast \\
\hline Region & 204 & 0 & 44 & 28 & 23 & 8 & 29 & 72 \\
Active & 75 & 0 & 23 & 14 & 12 & 3 & 7 & 16 \\
Inactive & 55 & 0 & 12 & 3 & 3 & 3 & 5 & 29 \\
$\begin{array}{c}\text { Test/Study } \\
\begin{array}{c}\text { \% Success of } \\
\text { Non-Testing/Study Sites }\end{array}\end{array}$ & 74 & 0 & 9 & 11 & 8 & 2 & 17 & 27 \\
\hline
\end{tabular}




\begin{tabular}{|c|c|c|c|c|c|c|c|c|c|}
\hline & Observations & Missing & None & Clogging & $\begin{array}{l}\text { Expired } \\
\text { Permit }\end{array}$ & $\begin{array}{c}\text { PWS } \\
\text { Conversion }\end{array}$ & Recovery & $\begin{array}{l}\text { Tested then } \\
\text { Abandoned }\end{array}$ & $\begin{array}{c}\text { Water } \\
\text { Quality/ } \\
\text { Arsenic }\end{array}$ \\
\hline Issues with ASR & 190 & 14 & 111 & 29 & 1 & 1 & 20 & 6 & 22 \\
\hline Active & 72 & 3 & 56 & 13 & 1 & 0 & 0 & 0 & 2 \\
\hline Inactive & 48 & 7 & 3 & 12 & 0 & 0 & 17 & 3 & 13 \\
\hline Test/Study & 70 & 4 & 52 & 4 & 0 & 1 & 3 & 3 & 7 \\
\hline $\begin{array}{c}\text { \% Success of } \\
\text { Non-Testing/Study Sites }\end{array}$ & & & $95 \%$ & $52 \%$ & $100 \%$ & N/A & $0 \%$ & $0 \%$ & $13 \%$ \\
\hline
\end{tabular}

\begin{tabular}{ccccccc}
\hline & Observations & Missing & Groundwater & Industrial & Reclaimed & Surface Runoff \\
\hline Water Source & 204 & 0 & 41 & 1 & 28 & 134 \\
Active & 75 & 0 & 13 & 1 & 0 & 52 \\
Inactive & 55 & 0 & 17 & 0 & 7 & 31 \\
Test/Study & 74 & 0 & 11 & $100 \%$ & $56 \%$ & 51 \\
$\begin{array}{c}\text { \% Success of } \\
\text { Non-Testing/Study Sites }\end{array}$ & & & $43 \%$ & & $63 \%$ \\
\hline
\end{tabular}

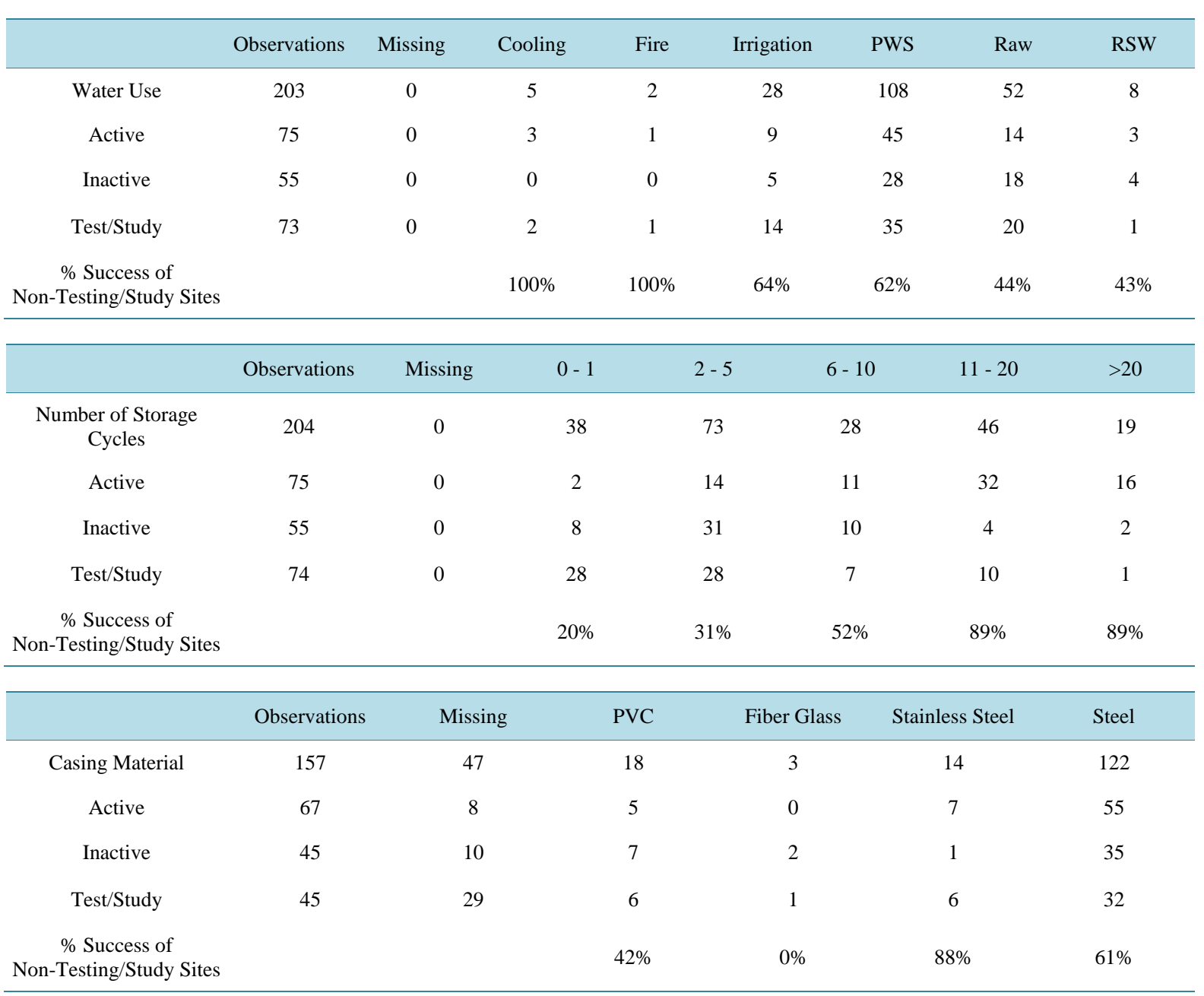




\begin{tabular}{|c|c|c|c|c|}
\hline & Observations & Missing & None & $\mathrm{T} \& \mathrm{P}$ \\
\hline T\&P Code & 185 & 19 & 180 & 5 \\
\hline Active & 74 & 1 & 73 & 1 \\
\hline Inactive & 53 & 2 & 50 & 3 \\
\hline Test/Study & 58 & 16 & 57 & 1 \\
\hline $\begin{array}{c}\text { \% Success of } \\
\text { Non-Testing/Study Sites }\end{array}$ & & & $59 \%$ & $25 \%$ \\
\hline
\end{tabular}

\begin{tabular}{|c|c|c|c|c|c|c|c|c|c|c|c|c|}
\hline & Observations & Missing & \multicolumn{2}{|c|}{ Alluvial } & Basalt & \multicolumn{2}{|c|}{ Carbonite } & Granite & Limestone & Sand & $\begin{array}{l}\text { Sand/Clay } \\
\text { Mixture }\end{array}$ & Sandstone \\
\hline $\begin{array}{l}\text { Injection Formation } \\
\text { Code }\end{array}$ & 185 & 19 & \multicolumn{2}{|l|}{61} & 14 & \multicolumn{2}{|c|}{2} & 1 & 62 & 16 & 12 & 17 \\
\hline Active & 70 & 5 & \multicolumn{2}{|l|}{31} & 4 & \multicolumn{2}{|c|}{1} & 0 & 13 & 9 & 6 & 6 \\
\hline Inactive & 51 & 4 & \multicolumn{2}{|l|}{12} & 1 & \multicolumn{2}{|c|}{1} & 0 & 26 & 4 & 1 & 6 \\
\hline Test/Study & 64 & 10 & \multicolumn{2}{|l|}{18} & 9 & \multicolumn{2}{|l|}{0} & 1 & 23 & 3 & 5 & 5 \\
\hline $\begin{array}{c}\text { \% Success of } \\
\text { Non-Testing/Study Sites }\end{array}$ & & & \multicolumn{2}{|c|}{$72 \%$} & $80 \%$ & \multicolumn{2}{|c|}{$50 \%$} & N/A & $33 \%$ & $69 \%$ & $86 \%$ & $50 \%$ \\
\hline & Observations & Missing & None & Alluvial & $\begin{array}{r}\text { Basa } \\
\mathrm{Mi}\end{array}$ & $\begin{array}{l}\text { alt/Clay } \\
\text { ixture }\end{array}$ & Bedrock & Clay & Dolomite & Limestone & Silt & $\begin{array}{l}\text { Shale/ } \\
\text { Sedimentary }\end{array}$ \\
\hline $\begin{array}{l}\text { Confinement Unit } \\
\text { Formation Code }\end{array}$ & 90 & 114 & 3 & 2 & & 14 & 3 & 30 & 13 & 13 & 7 & 5 \\
\hline Active & 32 & 43 & 1 & 1 & & 3 & 2 & 12 & 5 & 3 & 3 & 2 \\
\hline Inactive & 28 & 27 & 1 & 0 & & 2 & 0 & 11 & 3 & 6 & 3 & 2 \\
\hline Test/Study & 30 & 44 & 1 & 1 & & 9 & 1 & 7 & 5 & 4 & 1 & 1 \\
\hline $\begin{array}{c}\text { \% Success of } \\
\text { Non-Testing/Study Sites }\end{array}$ & & & $50 \%$ & $100 \%$ & & $60 \%$ & $100 \%$ & $52 \%$ & $63 \%$ & $33 \%$ & $50 \%$ & $50 \%$ \\
\hline
\end{tabular}

Table 3. Description of region categories for the ASR sites (developed by Bloetscher).

\begin{tabular}{|c|c|c|c|c|c|c|}
\hline & Basin Range & California & Mid-Atlantic & Mid-West & Pacific NW & SE Coast \\
\hline & Arizona & California & Delaware & Illinois & Idaho & Florida \\
\hline & Colorado & & New Jersey & Iowa & Oregon & North Carolina \\
\hline & Kansas & & New York & Minnesota & South Dakota & South Carolina \\
\hline \multirow[t]{4}{*}{ States } & New Mexico & & & Wisconsin & Washington & Tennessee \\
\hline & Nevada & & & & & Virginia \\
\hline & Texas & & & & & \\
\hline & Utah & & & & & \\
\hline
\end{tabular}


Table 4. Summary of logistic regression statistics predicting ASR success in the United States. Statistically significant coefficients are highlighted in yellow bold (95\% confidence). Coefficients that were not included in this analysis are denoted by the “-” sign.

\begin{tabular}{|c|c|c|c|c|c|c|c|c|c|c|c|}
\hline Missing values omitted for... & All & Issues & $\begin{array}{l}\text { Withdrawal } \\
\text { capacity }\end{array}$ & $\begin{array}{l}\text { In/out } \\
\text { ratio }\end{array}$ & $\begin{array}{l}\text { Well } \\
\text { depth }\end{array}$ & $\begin{array}{l}\text { Injection } \\
\text { horizon }\end{array}$ & $\begin{array}{l}\text { Casing } \\
\text { material }\end{array}$ & $\begin{array}{l}\text { Casing } \\
\text { diameter }\end{array}$ & $\begin{array}{l}\text { Injection } \\
\text { formation }\end{array}$ & Transmissivity & Confinement \\
\hline \multicolumn{12}{|l|}{ Model Statistics } \\
\hline Observations & 204 & 120 & 126 & 121 & 125 & 111 & 112 & 99 & 121 & 51 & 60 \\
\hline $\begin{array}{l}\text { Classification accuracy } \\
\text { (null model) }(\%)\end{array}$ & 57.7 & 59.5 & 58.3 & 59.8 & 57.9 & 58.0 & 59.3 & 56.0 & 57.4 & 57.7 & 52.5 \\
\hline $\begin{array}{l}\text { Classification accuracy } \\
\text { (with variables) }(\%)\end{array}$ & 67.7 & 90.1 & 69.3 & 69.7 & 68.3 & 71.4 & 69.0 & 69.0 & 72.1 & 61.5 & 77.0 \\
\hline $\begin{array}{c}\text { Improvement of } \\
\text { classification accuracy (\%) }\end{array}$ & 10 & 30.6 & 11 & 9.9 & 10.4 & 13.4 & 9.7 & 13.0 & 14.7 & 3.8 & 24.5 \\
\hline $\begin{array}{l}\text { P-value of Hosmer and } \\
\text { Lemeshow test [39] }\end{array}$ & 0.267 & 0.837 & 0.329 & 0.220 & 0.528 & 0.506 & 0.308 & 0.218 & 0.238 & 0.421 & 0.475 \\
\hline Cox \& Snell Pseudo R² [40] & 0.142 & 0.583 & 0.170 & 0.178 & 0.161 & 0.173 & 0.197 & 0.115 & 0.211 & 0.039 & 0.432 \\
\hline Nagelkerke Pseudo R ${ }^{2}$ [41] & 0.192 & 0.788 & 0.229 & 0.241 & 0.216 & 0.233 & 0.266 & 0.155 & 0.284 & 0.053 & 0.576 \\
\hline \multicolumn{12}{|l|}{$\begin{array}{l}\text { Regression Coefficients } \\
\text { (Log-Odds) }\end{array}$} \\
\hline Constant & 21.021 & -1.868 & -0.162 & -0.184 & -0.752 & -0.883 & -19.841 & -0.152 & -0.890 & -0.215 & -21.203 \\
\hline Region-Southeast Coast & -0.389 & 1.573 & -0.414 & -0.460 & 0.140 & 0.550 & 0.195 & 0.369 & -0.103 & 0.983 & -2.569 \\
\hline Region-California & 1.571 & 5.559 & 2.645 & 2.569 & 2.342 & 2.720 & 2.056 & 1.889 & 0.831 & 1.029 & 36.197 \\
\hline Region-Pacific Northwest & 0.182 & 3.737 & 0.320 & 0.088 & 0.802 & 1.300 & 0.537 & 0.530 & -22.309 & 0.390 & 29.670 \\
\hline Region-Basin Range & 0.713 & 3.277 & 0.634 & 0.757 & 1.003 & 2.066 & 1.031 & 1.050 & -0.030 & 1.124 & -0.413 \\
\hline Region-Mid-Atlantic & 1.588 & 1.710 & 1.567 & 1.459 & 1.897 & 2.534 & 2.239 & 2.157 & 0.462 & 0.590 & 18.767 \\
\hline Issue-Clogging & - & -4.490 & - & - & - & - & - & - & - & - & - \\
\hline $\begin{array}{l}\text { Issue-Water quality or arsenic } \\
\text { problems }\end{array}$ & - & -5.616 & - & - & - & - & - & - & - & - & - \\
\hline Issue-Expired permit & - & 18.236 & - & - & - & - & - & - & - & - & - \\
\hline Water source-groundwater & -21.476 & 3.263 & -0.289 & -0.469 & -0.411 & -0.997 & -20.048 & -0.503 & 0.244 & -0.270 & 5.097 \\
\hline Water source-reclaimed water & -21.102 & 3.871 & 0.133 & -0.349 & 0.077 & -0.776 & -19.139 & -0.038 & 0.394 & -0.833 & 4.658 \\
\hline Water source-surface runoff & -21.021 & 2.774 & 0.191 & -0.039 & -0.032 & -0.379 & -19.477 & 0.011 & 0.645 & -0.253 & 6.235 \\
\hline Withdrawal capacity (MGD) & - & - & 0.001 & -0.004 & - & - & - & - & - & - & - \\
\hline In/out ratio & - & - & - & -0.432 & - & - & - & - & - & - & - \\
\hline Well depth (ft) & - & - & - & - & 0.001 & 0.001 & 0.000 & - & - & - & - \\
\hline Injection horizon (ft) & - & - & - & - & - & -0.001 & - & - & - & - & -0.003 \\
\hline Casing material-steel & - & - & - & - & - & - & 38.840 & - & - & - & - \\
\hline Casing material-PVC & - & - & - & - & - & - & 38.116 & - & - & - & - \\
\hline Casing material-stainless steel & - & - & - & - & - & - & 39.562 & - & - & - & - \\
\hline Casing diameter (ft) & - & - & - & - & - & - & - & -0.028 & - & - & - \\
\hline Injection formation-sandstone & - & - & - & - & - & - & - & - & 0.203 & - & - \\
\hline Injection formation-sand & - & - & - & - & - & - & - & - & 1.097 & - & - \\
\hline Injection formation-limestone & - & - & - & - & - & - & - & - & -0.144 & - & - \\
\hline Injection formation-alluvial & - & - & - & - & - & - & - & - & 1.352 & - & - \\
\hline
\end{tabular}




\section{Continued}

\begin{tabular}{|c|c|c|c|c|c|c|c|c|c|c|c|}
\hline Injection formation-basalt & - & - & - & - & - & - & - & - & 24.090 & - & - \\
\hline $\begin{array}{l}\text { Injection formation-sand/clay } \\
\text { mixture }\end{array}$ & - & - & - & - & - & - & - & - & 1.702 & - & - \\
\hline Transmissivity (gpd/sf) & - & - & - & - & - & - & - & - & - & -0.215 & - \\
\hline Confinement-silt & - & - & - & - & - & - & - & - & - & - & 16.474 \\
\hline $\begin{array}{l}\text { Confinement-shale/sedimentary } \\
\text { mixture }\end{array}$ & - & - & - & - & - & - & - & - & - & - & 17.410 \\
\hline Confinement-clay & - & - & - & - & - & - & - & - & - & - & 20.179 \\
\hline Confinement-limestone & - & - & - & - & - & - & - & - & - & - & 19.141 \\
\hline Confinement-bedrock & - & - & - & - & - & - & - & - & - & - & 38.478 \\
\hline Confinement-dolomite & - & - & - & - & - & - & - & - & - & - & 21.010 \\
\hline Confinement-basalt/clay & - & - & - & - & - & - & - & - & - & - & -12.637 \\
\hline Confinement-alluvial & - & - & - & - & - & - & - & - & - & - & 4.298 \\
\hline
\end{tabular}

Table 5. Contingency tables of residual values (observed-expected) and Chi-Square test results comparing ASR success and each categorical variable of ASR sites. Statistically significant Chi-Square statistics are highlighted in yellow (95\% confidence).

\begin{tabular}{|c|c|c|c|c|c|c|c|c|c|c|c|c|c|c|c|c|c|c|c|c|c|c|c|c|c|c|}
\hline & & \multicolumn{24}{|c|}{ State of the ASR site } & \multirow{2}{*}{ Total } \\
\hline & & $\mathrm{AZ}$ & CA & $\mathrm{CO}$ & $\mathrm{DE}$ & FL & IA & ID & IL & KS & $\mathrm{MN}$ & NC & NJ & NM & NV & NY & OR & SC & SD & $\mathrm{TN}$ & $\mathrm{TX}$ & UT & VA & WA & WI & \\
\hline \multirow{3}{*}{$\begin{array}{c}\text { ASR } \\
\text { success }\end{array}$} & Active & 1.8 & 4.2 & -1.5 & 0.4 & -11.2 & 0.8 & 0.8 & -0.6 & 0.4 & 0.4 & 0.4 & 4.1 & -0.6 & 1.7 & -1.2 & 0.1 & 1.5 & -0.6 & -0.6 & 0.7 & 0.3 & -0.2 & -0.3 & -1.2 & 0 \\
\hline & Inactive & -1.8 & -4.2 & 1.5 & -0.4 & 11.2 & -0.8 & -0.8 & 0.6 & -0.4 & -0.4 & -0.4 & -4.1 & 0.6 & -1.7 & 1.2 & -0.1 & -1.5 & 0.6 & 0.6 & -0.7 & -0.3 & 0.2 & 0.3 & 1.2 & 0 \\
\hline & Total & 0 & 0 & 0 & 0 & 0 & 0 & 0 & 0 & 0 & 0 & 0 & 0 & 0 & 0 & 0 & 0 & 0 & 0 & 0 & 0 & 0 & 0 & 0 & 0 & 0 \\
\hline
\end{tabular}

$\chi_{c}^{2}=48.905$.

\begin{tabular}{|c|c|c|c|c|c|c|c|c|}
\hline & & \multicolumn{6}{|c|}{ Region of ASR site } & \multirow{2}{*}{ Total } \\
\hline & & Basin Range & California & Mid-Atlantic & Mid-West & Pacific NW & SE Coast & \\
\hline \multirow{3}{*}{$\begin{array}{c}\text { ASR } \\
\text { success }\end{array}$} & Active & 2.8 & 4.2 & 3.3 & -0.5 & 0.1 & -10.0 & 0 \\
\hline & Inactive & -2.8 & -4.2 & -3.3 & 0.5 & -0.1 & 10.0 & 0 \\
\hline & Total & 0 & 0 & 0 & 0 & 0 & 0 & 0 \\
\hline
\end{tabular}

$\chi_{c}^{2}=17.399$.

\begin{tabular}{|c|c|c|c|c|c|c|c|c|}
\hline & & \multicolumn{6}{|c|}{ Issues with ASR site } & \multirow[b]{2}{*}{ Total } \\
\hline & & None & Clogging & Expired Permit & Recovery & $\begin{array}{l}\text { Tested then } \\
\text { abandoned }\end{array}$ & $\begin{array}{l}\text { Water quality/ } \\
\text { arsenic }\end{array}$ & \\
\hline \multirow{3}{*}{$\begin{array}{c}\text { ASR } \\
\text { success }\end{array}$} & Active & 20.6 & -2 & 0.4 & -10.2 & -1.8 & -7 & 0 \\
\hline & Inactive & -20.6 & 2 & -0.4 & 10.2 & 1.8 & 7 & 0 \\
\hline & Total & 0 & 0 & 0 & 0 & 0 & 0 & 0 \\
\hline
\end{tabular}

$\chi_{c}^{2}=74.913$.

\begin{tabular}{|c|c|c|c|c|c|c|}
\hline & & \multicolumn{4}{|c|}{ Water Source } & \multirow{2}{*}{ Total } \\
\hline & & Groundwater & Industrial & Reclaimed & Surface Runoff & \\
\hline \multirow{3}{*}{$\begin{array}{c}\text { ASR } \\
\text { success }\end{array}$} & Active & -4.3 & 0.4 & -0.2 & 4.1 & 0 \\
\hline & Inactive & 4.3 & -0.4 & 0.2 & -4.1 & 0 \\
\hline & Total & 0 & 0 & 0 & 0 & 0 \\
\hline
\end{tabular}

$\chi_{c}^{2}=4.117$. 


\begin{tabular}{|c|c|c|c|c|c|c|c|c|}
\hline & & \multicolumn{6}{|c|}{ Water Use } & \multirow{2}{*}{ Tota } \\
\hline & & Cooling & Fire & Irrigation & PWS & Raw & RSW & \\
\hline \multirow{3}{*}{$\begin{array}{c}\text { ASR } \\
\text { success }\end{array}$} & Active & 1.3 & 0.4 & 0.9 & 2.9 & -4.5 & -1.0 & 0 \\
\hline & Inactive & -1.3 & -0.4 & -0.9 & -2.9 & 4.5 & 1.0 & 0 \\
\hline & Total & 0 & 0 & 0 & 0 & 0 & 0 & 0 \\
\hline
\end{tabular}

$\chi_{c}^{2}=6.829$.

\begin{tabular}{|c|c|c|c|c|c|c|c|}
\hline & & \multicolumn{5}{|c|}{ Number of Storage Cycles } & \multirow{2}{*}{ Total } \\
\hline & & $0-1$ & $2-5$ & $6-10$ & $11-20$ & $>20$ & \\
\hline \multirow{3}{*}{$\begin{array}{c}\text { ASR } \\
\text { success }\end{array}$} & Active & -3.8 & -12.0 & -1.1 & 11.2 & 5.6 & 0 \\
\hline & Inactive & 3.8 & 12.0 & 1.1 & -11.2 & -5.6 & 0 \\
\hline & Total & 0 & 0 & 0 & 0 & 0 & 0 \\
\hline
\end{tabular}

$\chi_{c}^{2}=40.621$.

\begin{tabular}{|c|c|c|c|c|c|c|}
\hline & & \multicolumn{4}{|c|}{ Casing Material } & \multirow{2}{*}{ Total } \\
\hline & & PVC & Fiber Glass & Stainless Steel & Steel & \\
\hline \multirow{3}{*}{$\begin{array}{c}\text { ASR } \\
\text { success }\end{array}$} & Active & -2.2 & -1.2 & 2.2 & 1.2 & 0 \\
\hline & Inactive & 2.2 & 1.2 & -2.2 & -1.2 & 0 \\
\hline & Total & 0 & 0 & 0 & 0 & 0 \\
\hline
\end{tabular}

$\chi_{c}^{2}=7.236$.

\begin{tabular}{cccccc}
\hline & & T\&P Code & Total \\
\cline { 3 - 5 } & & None & T\&P & 0 \\
ASR & Active & 1.3 & -1.3 & 0 \\
success & Inactive & -1.3 & 1.3 & 0 \\
& Total & 0 & 0 & 0 \\
\hline
\end{tabular}

$\chi_{c}^{2}=1.880$.

\begin{tabular}{cccccccccc}
\hline & & \multicolumn{9}{c}{ Injection Formation Code } & & \\
\cline { 3 - 7 } & & Alluvial & Basalt & Carbonite & Limestone & Sand & $\begin{array}{c}\text { Sand/Clay } \\
\text { Mixture }\end{array}$ & Sandstone & Total \\
ASR & Active & 6 & 1 & 0 & -10 & 1 & 2 & -1 & 0 \\
success & Inactive & -6 & -1 & 0 & 10 & -1 & -2 & 1 & 0 \\
& Total & 0 & 0 & 0 & 0 & 0 & 0 & 0 & 0 \\
\hline
\end{tabular}

$\chi_{c}^{2}=17.470$.

\begin{tabular}{ccccccccccccc}
\hline & & \multicolumn{7}{c}{ Confinement Unit Formation Code } \\
\cline { 3 - 11 } & & None & Alluvial & $\begin{array}{c}\text { Basalt/Clay } \\
\text { Mixture }\end{array}$ & Bedrock & Clay & Dolomite & Limestone & Silt & Shale/Sedimentary & Total \\
\hline \multirow{2}{*}{ ASR } & Active & -0.1 & 0.5 & 0.3 & 0.9 & -0.3 & 0.7 & -1.8 & -0.2 & -0.1 & 0 \\
& Inactive & 0.1 & -0.5 & -0.3 & -0.9 & 0.3 & -0.7 & 1.8 & 0.2 & 0.1 & 0 \\
& Total & 0 & 0 & 0 & 0 & 0 & 0 & 0 & 0 & 0 & 0 \\
\hline
\end{tabular}

$\chi_{c}^{2}=4.497$. 
contingency table between the two categorical variables of interest, where the expected values are defined as:

$$
E_{i j}=\frac{\left(R_{i}\right)\left(C_{i}\right)}{n}
$$

where $R_{i}$ is the row total corresponding to row $i, C_{i}$ is the column total corresponding to column $i$ and $n$ is the total number of observations. The association between two categorical variables can be assessed using the Chi-Square statistic $\left(\chi_{c}^{2}\right)$, which can be defined as:

$$
\chi_{c}^{2}=\sum_{i}^{r} \sum_{j}^{c} \frac{\left(O_{i j}-E_{i j}\right)^{2}}{E_{i j}}
$$

In addition to Chi-Square tests, logistic regression was performed to identify explanatory variables of ASR success in the United States. Logistic regression is a probabilistic statistical technique that uses a logistic function to predict the outcome of a dichotomous variable based on one or more predictor variables. A logistic function $F(t)$ can be defined as:

$$
F(t)=\frac{e^{t}}{e^{t}+1}=\frac{1}{1+e^{-t}}
$$

where $t$ is a linear function of at least one or more explanatory variables. Equation (1) can also be rewritten as:

$$
F(x)=\frac{1}{1+e^{-\beta_{0}+\beta_{1} x_{1}+\cdots}}
$$

where $\beta_{0}$ is the intercept from the linear regression equation and $\beta_{1}$ is a regression coefficient for one of the explanatory variables. Equation (2) can be interpreted as the probability of $F(x)$ equaling a value of 1 (rather than 0 ). One can also define the inverse of the logistic function, $G(x)$, which is also called the logit, log-odds or natural logarithm of the odds function:

$$
G(x)=\ln \frac{F(x)}{1-F(x)}=\beta_{0}+\beta_{1} x_{1}+\cdots
$$

The $\beta$ coefficients in logistic regression are estimated using a maximum likelihood estimator.

We used success (1) or failure (0) of ASR sites in the United States, as the dichotomous dependent variable. A number of different logistic regression models were tested by varying the sets of continuous (Table 2(a)) and categorical (Table 2(b)) explanatory variables; the model sets were driven by the exploratory questions and missing data. Variables shown to be measuring similar phenomena (i.e., based on physical characteristics and correlation analysis) such as well depth and casing depth, were not used together in the tested models to avoid multi-co-linearity and other artifacts. Seven continuous explanatory variables were used in the logistic regression analyses: estimated start date, withdrawal capacity, in/out water volume ratio, depth of well, injection horizon, casing diameter and transmissivity. Six categorical variables were transformed into dummy variables and then used as explanatory variables in the logistic regression analyses: region, issues with ASR program, water source, casing material, injection formation code and confinement unit formation code.

\section{Results and Discussion}

The state with the largest numbers of ASR programs are Florida (54), followed by California, New Jersey, Arizona and Oregon (see Figure 3). However, the presence of ASR sites is not necessarily indicative for success of ASR projects. For example, in Florida, over half the sites are no longer active or have wells that are no longer used (see Figure 4). Other states that have large numbers of inactive sites are California and Arizona. With the elimination of inactive and test sites, there are only 22 active ASR sites (as compared to the total of 54 ASR sites) in Florida. Figure 5 shows the number of sites in the test phase by state. It should be noted that most of the inactive ASR sites have only one well, although a few outliers with many wells skews the average number of wells per site in Table 1. There also appears to be a slowing of ASR development after a marked rise in the 1990s and early 2000s (see Figure 6 [3]). 


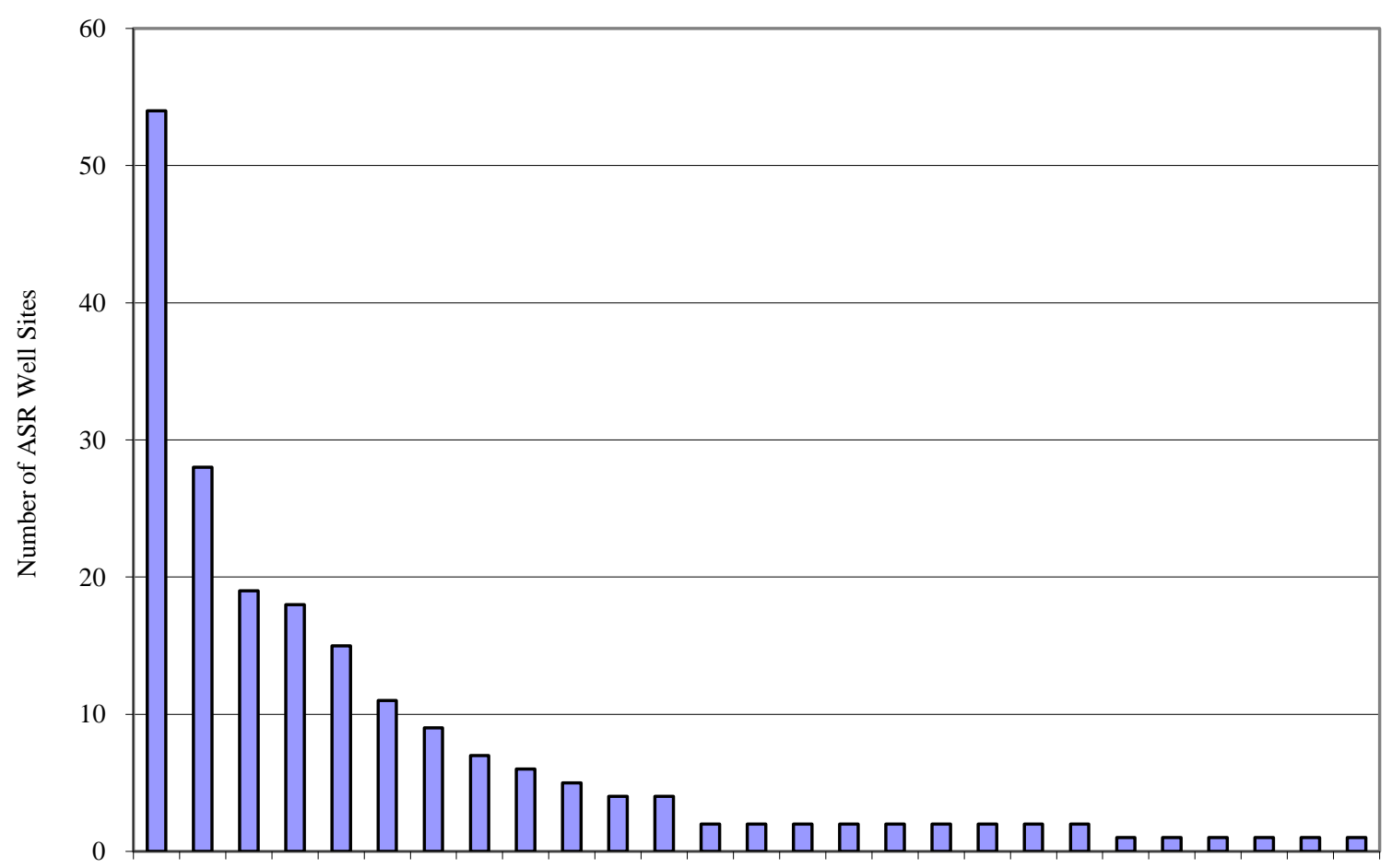

FL CA NJ AZ OR SC WA CO TX NV IA UT DE GA ID KS NC NY VA WI WY IL MNNM OK SD TN State

Figure 3. Number of ASR wells by state.

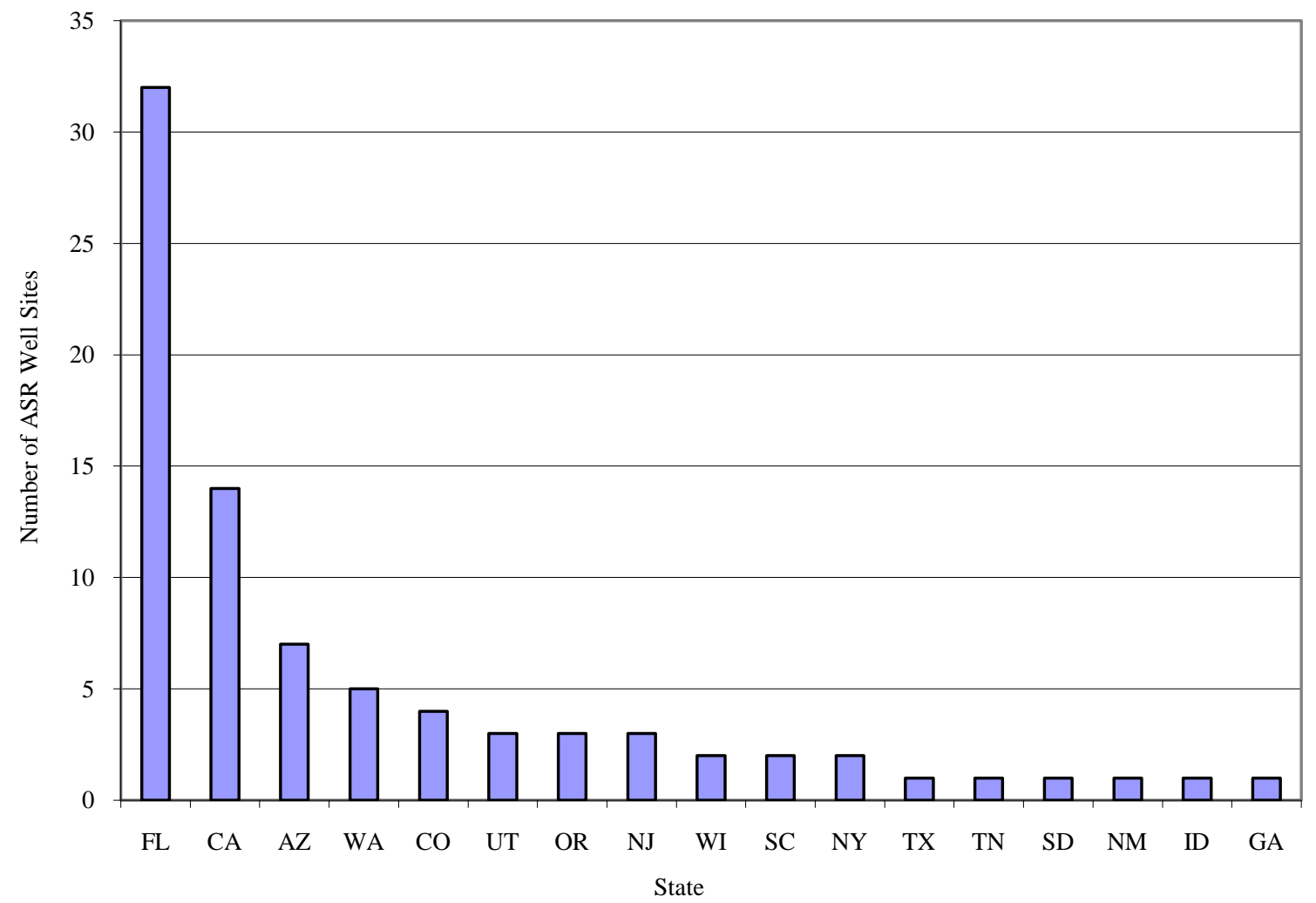

Figure 4. Systems not in operation by states. 


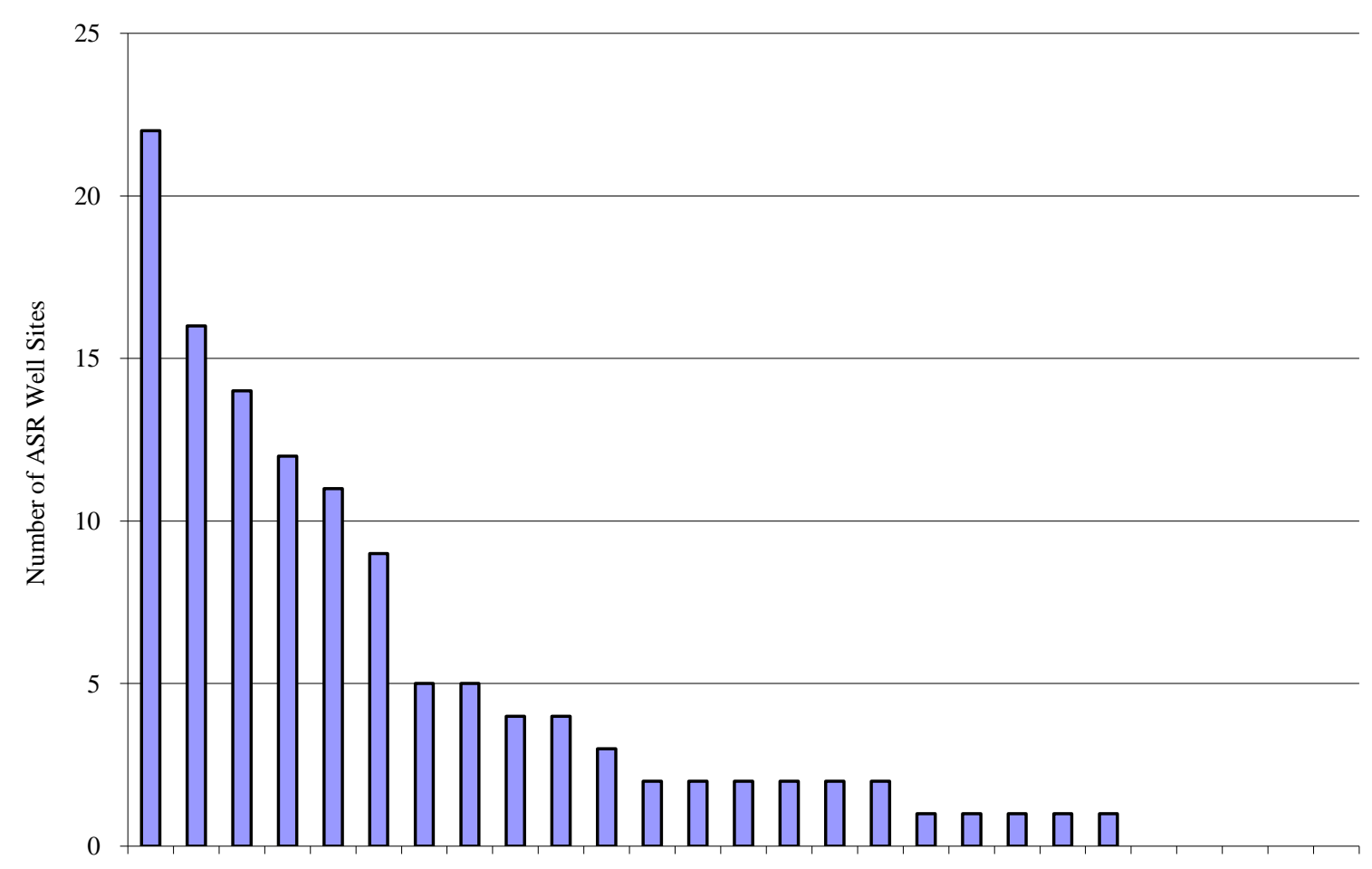

FL NJ CA OR AZ SC TX NV WA IA CO DE KS NC VA WI WY UT GA ID IL OK NY MNNM SD TN State

Figure 5. Net systems in operation, investigation or test phase by state.

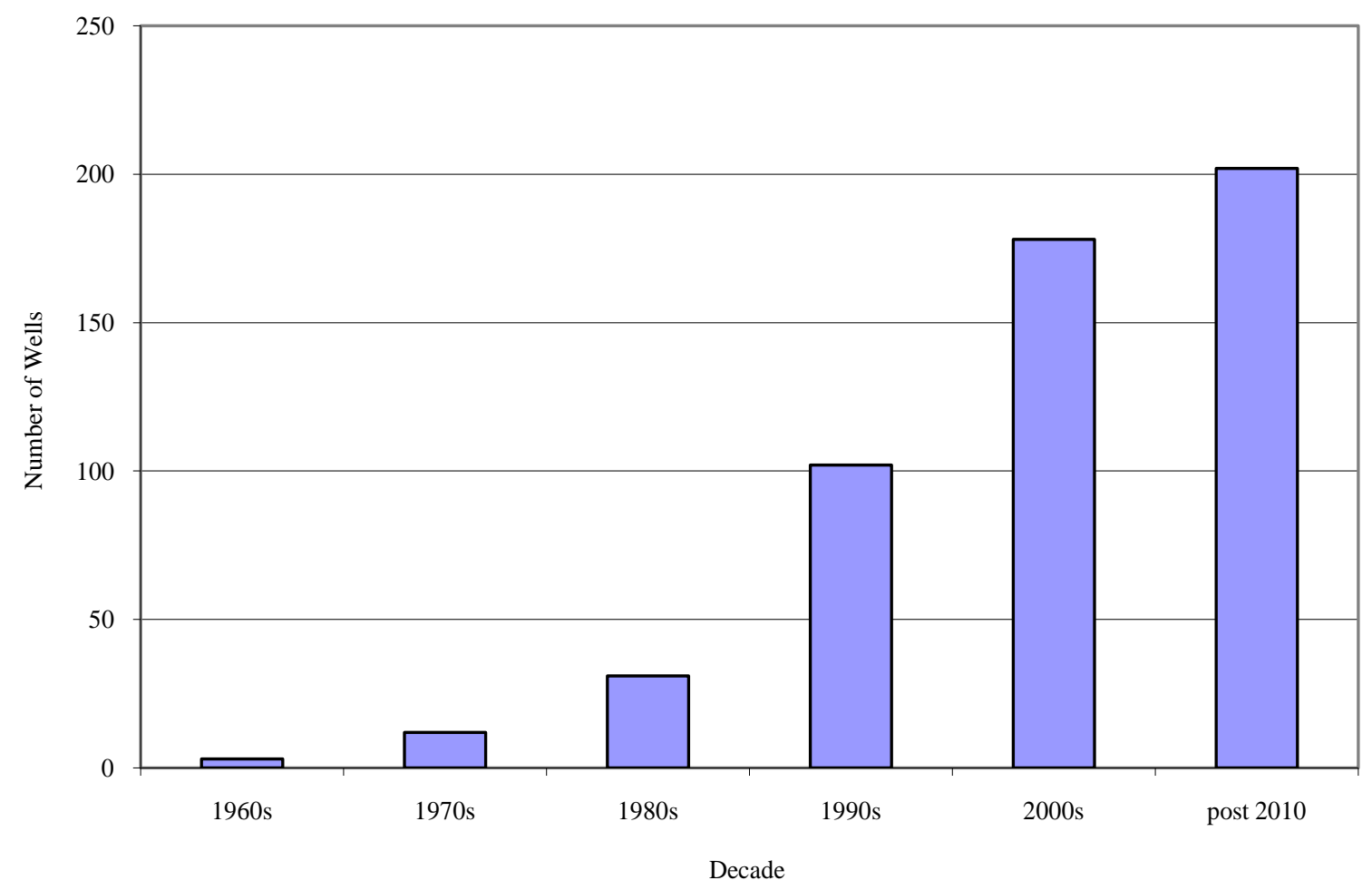

Figure 6. Growth of ASR investigations by decade. 
With 95\% confidence, chi-square tests results indicate that there are no statistical differences between ASR program status (active $\&$ inactive) and the following categorical variables: water source, water use, casing material, T\&P code and confinement unit formation code. With 95\% confidence, regression findings suggest that success rate is higher for ASR sites located in California, the Pacific Northwest, and Rocky Mountain/Plains.

Chi-square tests results indicate that there are statistically differences between ASR program status (active and inactive) and the following categorical variables: state, region, operational issues, storage cycles and injection formation code. Success rate is lower for ASR sites with deeper wells, clogging problems and water quality/ arsenic issues; clogging and water quality are operational issues. Based on these observations, the authors re-examined the data in order to discern the causes of operational issues with ASR systems, focusing primarily on the inactive wells, and those that have indicated issues with operations.

Table 6 outlines the information related to wells that are inactive. Table 7 outlines issues that have been raised with respect to active wells. Among the wells that are inactive, 10 wells were affected by water quality issues. Five of those are related to arsenic in Florida [4] [5], and four are associated with arsenic, manganese, iron or a combination of metals (e.g., in Wisconsin [6]). Two additional wells are related to radon in the west [7] [8]. The arsenic releases are all from limestone aquifers, and are associated with a range of injected water quality. Nevertheless, change in the geochemistry induced by injected water may lead to the release of arsenic from the geologic formations. Among the wells that are still active but not operational, a total of 6 wells in Florida are all releasing arsenic from limestone formations. One of these wells (i.e., Bradenton), is actively looking at deploying ion exchange as a means to deal with arsenic in the recovered water. Recently, U.S. EPA and the state of Florida have entered into a memorandum of understanding on permitting issues associated with arsenic in recovered water [9].

Clogging is another ongoing problem at many sites. Eleven sites were identified as being abandoned as a result of clogging. Although 18 other sites are still active, they are associated with ongoing clogging issues. Among the inactive wells, 9 of them are injecting surface water into alluvial formations. The mechanism for clogging was not explicitly identified in these cases, but biofouling, particulates and geochemistry are all potential factors because of the organics in the water sources. Gin [10] [11] and Walker [12] reported that the clogging of the Phoenix wells (one using surface water and two injecting reclaimed water) got so bad that clearing the wells was required every three days, which led to the abandonment of the systems. Clogging is an issue at 18 active sites for which 13 of them are alluvial formations with a majority of them injecting surface water. Most of these sites are located in the west and they all have steel casings which may contribute to the potential for biofouling [13].

Low recovery of injected water has derailed ASR programs at 17 sites. The majority of these sites are in Colorado or Florida. Boomgaard [14] indicated that recovery was low in the abandoned Colorado wells. In addition, it was easier and cheaper to obtain new water rights from west of the Continental divide than to keep the ASR program going.

As for the Florida sites that are no longer active, low recovery is mostly associated with sites that attempted to inject potable water into limestone formations containing brackish groundwater. Many are in southeast Florida where only the Boynton Beach wells have been successful to date [5]. Bloetscher and Muniz [15] suggest that the percent recovery would improve with lesser recovery from the well, but the clients were discouraged by the low recovery and hence abandoned the project. The CERP projects partially fall into this category of low recovery (although arsenic was a bigger issue). The Dalton, Georgia well was a private project that injected into granite [16] [17]. It is the only granite well and relies of fissure for success. Given north Georgia is known for its tight geologic formations and limited fissures, it is not surprising that the project was abandoned due to a lack of recovery.

Table 6 and Table 7 indicate that well problems leading to inactivity or abandonment can be broken into three groups: 1) physical and chemical clogging or biological fouling, 2) inability to adequately recover injected water, 3) water quality changes which include arsenic, metals mobilization and trihalomethanes [5] [6] [18]. Each of these issues are outlined in the following sections.

\subsection{Well Clogging}

Well clogging was reported as one of the major operational problems with ASR wells [19]. Clogging was mentioned as a problem at 29 inactive sites of the 204 ASR sites, including 11 as the main reason for why the wells 
Table 6. Characteristics of wells that are no longer active sorted by reason inactive.

\begin{tabular}{|c|c|c|c|c|c|c|c|c|}
\hline State & Project/Location & $\begin{array}{l}\text { Est Start } \\
\text { Date }\end{array}$ & $\begin{array}{l}\text { Number of } \\
\text { abandoned wells } \\
\text { in the project }\end{array}$ & $\begin{array}{l}\text { Reason To } \\
\text { Abandon }\end{array}$ & $\begin{array}{l}\text { Injection } \\
\text { Cap }\end{array}$ & $\begin{array}{l}\text { Formation } \\
\text { Type }\end{array}$ & $\begin{array}{l}\text { Casing } \\
\text { Material }\end{array}$ & $\begin{array}{l}\text { Source of } \\
\text { Water }\end{array}$ \\
\hline FL & Punta Gorda-Shell Creek & $1996 / 2002$ & 4 & Arsenic & 1.4 & Limestone & $\mathrm{S}$ & Drinking Water \\
\hline FL & Lee County, Olga WTP & 2001 & 1 & Arsenic & 1 & Limestone & & GW \\
\hline FL & CERP St Lucie & 2001 & 1 & Arsenic & 5 & Limestone & PVC & Raw GW \\
\hline FL & Collier County North & 2006 & 1 & Arsenic & 1 & Limestone & FG & Reclaimed \\
\hline FL & $\begin{array}{l}\text { CERP Taylor Creek } \\
\text { SFWMD (Cl 61k) }\end{array}$ & 1989 & 1 & Arsenic & $0.3-0.5$ & Limestone & $S$ & Surface \\
\hline FL & $\begin{array}{l}\text { NW Hillsborough } \\
\text { (WQ deter) }\end{array}$ & 2003 & 1 & WQ Deterioration & 2 & Limestone & $\mathrm{S}$ & Reclaimed \\
\hline SC & Myrtle Beach & 1987 & 1 & WQ Deterioration & 0.5 & Limestone & S & Surface Water \\
\hline WI & Oak Creek & 1998 & 2 & Metals Mobilizatin & 1 & Sandstone & S & Surface \\
\hline WI & Green Bay Utlility & 1998 & 1 & Metals Mobilizatin & 1 & $\begin{array}{l}\text { Sandstone/ } \\
\text { Limestone }\end{array}$ & $\mathrm{S}$ & Surface \\
\hline $\mathrm{TN}$ & $\begin{array}{l}\text { Memphis Light } \\
\text { Gas and Water }\end{array}$ & 1995 & 1 & WQ Deterioration & 0.8 & Sand & S/SS & Ground \\
\hline OR & $\begin{array}{l}\text { Clackamas River } \\
\text { Water (radon) }\end{array}$ & 2000 & 1 & Radon & 0.5 & Basallt & S & Surface \\
\hline WA & $\begin{array}{c}\text { Seattle Public Utilities } \\
\text { Highline }\end{array}$ & 1989 & 3 & Radon & 1 & Alluvial & SS SC & Surface \\
\hline NY & Bay Park (clog) & 1968 & 1 & Clogging & 0.3 & Alluvial & $S$ & PWS \\
\hline $\mathrm{AZ}$ & $\begin{array}{c}\text { Phoenix 1-Salt River } \\
\text { Project }\end{array}$ & 1995 & 8 & Clogging & 1 & Alluvial & PVC & Raw Surface \\
\hline $\mathrm{AZ}$ & Phoenix 2-Tormanto & 2004 & 2 & Clogging & 2.5 & Alluvial & PVC & Raw Surface \\
\hline $\mathrm{AZ}$ & North Scottsdale & 2006 & 2 & Clogging & 0.5 & Alluvial & $S$ & Raw Surface \\
\hline $\mathrm{AZ}$ & Peoria-Stone Street & 1963 & 1 & Clogging & 1.6 & Alluvial & $\mathrm{S}$ & Raw Surface \\
\hline $\mathrm{AZ}$ & Phoenix 3-Cave Creek & 2007 & 2 & Clogging & 1.5 & Alluvial & PVC & Reclaimed \\
\hline NY & $\begin{array}{l}\text { East Meado Reclaimed } \\
\text { Water (Clog) }\end{array}$ & 2000 & 5 & Clogging & 0.5 & Sand & & Reclaimed \\
\hline UT & $\begin{array}{c}\text { Salt Lake City/High } \\
\text { Plains }\end{array}$ & 2003 & 40 & Clogging & 0.6 & Alluvial & & Surface \\
\hline OR & $\begin{array}{l}\text { Portland Water Bureau } \\
\text { (Shore) }\end{array}$ & 2003 & 2 & Clogging & 1.1 To 4 & $\begin{array}{l}\text { Alluvial/ } \\
\text { Sandstone }\end{array}$ & S & Surface \\
\hline FL & Ft. Myers Winkler & 1998 & 1 & Clogging & 1 & Limestone & & Surface \\
\hline FL & $\begin{array}{l}\text { Lee County, } \\
\text { North Reservoir }\end{array}$ & 2000 & 1 & Clogging & 1 & Limestone & & Surface \\
\hline FL & Marathon & 1990 & 1 & Recovery & 0.25 & Limestone & S & PWS GW \\
\hline FL & Sunrise Springtree & 2002 & 1 & Recovery & 1 & Limestone & $\mathrm{S}$ & PWS GW \\
\hline
\end{tabular}




\section{Continued}

\begin{tabular}{|c|c|c|c|c|c|c|c|c|}
\hline FL & Miami Beach & 1998 & 1 & Recovery & & Sandstone & S & PWS GW \\
\hline FL & Deerfield Beach, City of & 2001 & 1 & Recovery & 1.3 & Limestone & S/PVC & PWS GW \\
\hline FL & Lee County, Corkscrew & 1996 & 6 & Recovery & 0.65 & Limestone & S & PWS GW \\
\hline FL & Delray Beach & 1996 & 1 & Recovery & 0.4 & Sandstone & S & PWS GW \\
\hline FL & Broward County & 1998 & 1 & Recovery & $0.8-1.6$ & Limestone & S & Raw GW \\
\hline FL & Fort Lauderdale Fiveash & 1999 & 1 & Recovery & 0.5 & Limestone & S & Raw GW \\
\hline FL & $\begin{array}{l}\text { Miami-Dade Water \& } \\
\text { Sewer Dept. NW }\end{array}$ & 1998 & 3 & Recovery & 5 & Limestone & S & Raw GW \\
\hline FL & SFWMD Jupiter & 1974 & 1 & Recovery & 5 & Limestone & S & Raw GW \\
\hline $\mathrm{CO}$ & $\begin{array}{l}\text { East Cherry Creek Valley } \\
\text { Water District and } \\
\text { Sanitation/Willows Water } \\
\text { District acq } 2001\end{array}$ & 1990 & 4 & Recovery & & Limestone & S & Surface \\
\hline FL & West Palm Beach & 1997 & 1 & Recovery & 3 & Limestone & S & Surface \\
\hline $\mathrm{CO}$ & Castle Pines North AR4 & 1999 & 1 & Recovery & 0.5 & Sandstone & S & Surface \\
\hline $\mathrm{CO}$ & $\begin{array}{l}\text { Parker Water and } \\
\text { Sanitation District }\end{array}$ & 1996 & 1 & Recovery & & Sandstone & S & Surface \\
\hline $\mathrm{CO}$ & $\begin{array}{l}\text { Colorado Springs Utilitie } \\
\text { Park Forest/Donata }\end{array}$ & 2004 & 2 & Recovery & $0.7,0.1$ & Sandstone & S & Surface \\
\hline TX & Laredo & 1996 & 4 & Recovery & 0.2 & Sandstone & S & Surface \\
\hline CA & Camarillo & 1979 & 2 & Recovery & 0 & & & Surface \\
\hline NM & Alamogordo & 1996 & 1 & Study Only & 0.8 & Limestone & $\mathrm{S}$ & Ground \\
\hline
\end{tabular}

Table 7. Characteristics of wells that are still active, but reported issues sorted by reason inactive.

\begin{tabular}{|c|c|c|c|c|c|c|c|c|c|}
\hline State & Project/ Location & $\begin{array}{l}\text { Est Start } \\
\text { Date }\end{array}$ & $\begin{array}{c}\text { In } \\
\text { Development }\end{array}$ & $\begin{array}{c}\text { Number of } \\
\text { abandoned } \\
\text { wells in the } \\
\text { project }\end{array}$ & $\begin{array}{c}\text { Reason to } \\
\text { abandon }\end{array}$ & $\begin{array}{l}\text { injection } \\
\text { Cap }\end{array}$ & $\begin{array}{l}\text { Formation } \\
\text { type }\end{array}$ & $\begin{array}{l}\text { Casing } \\
\text { Material }\end{array}$ & $\begin{array}{l}\text { Source } \\
\text { of water }\end{array}$ \\
\hline FL & Bonita Springs San Carlos & 2000 & Study-Ab & 1 & Arsenic & 1 & Limestone & $\mathrm{S}$ & $\begin{array}{c}\text { Drinking } \\
\text { Water }\end{array}$ \\
\hline FL & Sanford & 2008 & Test & 0 & Arsenic & 1 & Limestone & $\mathrm{S}$ & PW \\
\hline FL & Englewood & 1998 & Op & 1 & Arsenic & 2.2 & Carbonate & $\mathrm{S}$ & Reclaimed \\
\hline FL & CERP Hillsboro Basin & 2001 & Study & 1 & Arsenic & 5 & Limestone & Pvc & Surface \\
\hline FL & Bradenton-Reservoir & 2007 & Hold & 1 & Arsenic & 3 & Limestone & & Surface \\
\hline FL & CERP Lake Okeechobee & 2004 & Test & 1 & Arsenic & 9.4 & Limestone & $\mathrm{S}$ & $\begin{array}{l}\text { Surface } \\
\text { Water }\end{array}$ \\
\hline CA & $\begin{array}{c}\text { Antelope Valley Los } \\
\text { Angeles PWD }\end{array}$ & 1994 & Op & 2 & Clogging & 1 & Alluvial & $\mathrm{S}$ & Ground \\
\hline
\end{tabular}




\section{Continued}

\begin{tabular}{|c|c|c|c|c|c|c|c|c|c|}
\hline UT & $\begin{array}{l}\text { Cachw/Leamington Town } \\
\text { Culinary Water Well }\end{array}$ & 2008 & Study Ex Well & 1 & Clogging & 1.3 & Limestone & S & Ground \\
\hline NJ & $\begin{array}{c}\text { Evesham Township } \\
\text { Municipal Utilities } \\
\text { Authority (They are } \\
\text { abandoning } 1 \text { well and } \\
\text { drilling another } 50 \mathrm{ft} \text { away) }\end{array}$ & 1996 & Op & 1 & Clogging & 0.5 & Sand/Clay & S & Ground \\
\hline $\mathrm{AZ}$ & Gilbert & 2005 & Op & 1 & Clogging & 2 & Alluvial & S & Reclaimed \\
\hline $\mathrm{AZ}$ & Chandler Intel & 2005 & Op & 3 & Clogging & & Alluvial & & $\begin{array}{l}\text { River Wa- } \\
\text { ter }\end{array}$ \\
\hline CA & $\begin{array}{l}\text { Victorville (Victor Valley } \\
\text { Water District) }\end{array}$ & 2006 & Op & 4 & Clogging & 0.8 & Alluvial & Epoxy Ss & Surface \\
\hline CA & $\begin{array}{l}\text { Valley Water Company } \\
\text { Foothills/Monk Hill Basin }\end{array}$ & 1994 & Op & 0 & Clogging & 1 & Alluvial & S & Surface \\
\hline NV & $\begin{array}{c}\text { Washoe County (Truckee) } \\
\text { gravity flow }\end{array}$ & 2008 & Op & 2 & Clogging & 0.2 & Alluvial & S & Surface \\
\hline UT & $\begin{array}{l}\text { Jordan Valley Water } \\
\text { District (retro wells) }\end{array}$ & 1994 & Op & 18 & Clogging & 0.7 & Alluvial & S & Surface \\
\hline OK & $\begin{array}{l}\text { Okahoma City, Blaine } \\
\text { Recharge Demo Project }\end{array}$ & 1972 & Pilot & 5 & Clogging & 1 & Alluvial & S & Surface \\
\hline CA & $\begin{array}{c}\text { Elsinore Valley Municipal } \\
\text { Water District }\end{array}$ & 2005 & Op & 0 & Clogging & 2 to 3 & Alluvial & S W Screen & Surface \\
\hline CA & $\begin{array}{l}\text { Pleasanton, } \\
\text { Zone } 7 \text { Livermore }\end{array}$ & 1998 & Op & 4 & Clogging & 1.7 & Alluvial & Ss & Surface \\
\hline CA & Tracy, City of & 2004 & Test & 1 & Clogging & 1.5 & Alluvial & Ss & Surface \\
\hline CA & Oxnard, City of & 2003 & Test/Ab & 5 & Clogging & 9 & Alluvial & Ss & Surface \\
\hline OR & Baker City, City of & 2004 & Op & 0 & Clogging & 1.1 & & S & Surface \\
\hline OR & Pendleton, City of & 2003 & Test & 2 & Clogging & 1.7 & Basallt & S & $\begin{array}{l}\text { Surface } \\
\text { Via Rbf }\end{array}$ \\
\hline NV & N Las Vegas & 1989 & Op & 6 & Clogging & 1 & Alluvial & S & $\begin{array}{l}\text { Varies, } \\
\text { Inc Reuse }\end{array}$ \\
\hline ID & Boise, United Water & 1991 & Op & 1 & Clogging & 0.3 & Basalt & S & \\
\hline CA & $\begin{array}{l}\text { Sonoma County Water } \\
\text { Agency-Santa Rosa ASR } \\
1 \text { aband to std wll }\end{array}$ & 2004 & Test & 1 & $\begin{array}{l}\text { Conv To } \\
\text { Well }\end{array}$ & 0.8 & Alluvial & S & Surface \\
\hline CA & East Bay MUD & 1997 & Test & 1 & Mn, Thm & $3759 \mathrm{~m}^{3} / \mathrm{d}$ & & S & Surface \\
\hline CA & Pasadena, City of (thm) & 1992 & Op & 3 & Thm & 1.2 & Alluvial & S & Surface \\
\hline WA & Yakima & 2002 & Test & 1 & Thm & 1.7 & Basalt & & PWS \\
\hline GA & $\begin{array}{c}\text { Dalton (carpet } \\
\text { industry-small test wells } \\
\text { only-no formation) }\end{array}$ & 2007 & Test & 5 & Recovery & & Granite & & Raw GW \\
\hline FL & PBC 3 & 1998 & Test & 1 & Recovery & 1 & Sandstone & S & Raw GW \\
\hline FL & PBC System 9 Hillsboro & 2003 & Test & 1 & Recovery & 5 & Limestone & $\mathrm{S}$ & Surface \\
\hline
\end{tabular}


are no longer active. All these wells were constructed with steel casings and most of them were using surface water as their source water, which makes particulate clogging and biofouling prime suspects of operational problems. As mentioned earlier, there are three types of clogging: particulate (or mechanical), chemical, and biological, along with combinations thereof. Mechanical clogging problems result from suspended solids clogging the pores of the receiving formation, well screens, and/or gravel packs. The Total Suspended Solid (TSS) concentration of the injected water, often characterized by turbidity, represents the most influential factor in determining the potential for this form of clogging, even when other factors may contribute to well clogging. Pyne et $a l$. [20] reported that it is rare to find an ASR well that will not clog if the injected water has high TSS. Pitt and Magenheimer [21] suggested that TSS concentrations at $0.5 \mathrm{mg} / \mathrm{L}$ to $1.0 \mathrm{mg} / \mathrm{L}$ can already cause clogging of a porous-media formation (e.g., an alluvial formation). Pyne [22] indicated that significant loss in recharge rates for most wells when the injected water are associated with TSS concentrations as low as $2.0 \mathrm{mg} / \mathrm{L}$. Despite the best efforts of operations, periodic clogging of the injection well is expected, as suspended solids reduce permeability and transmissivity of the formation in the zone surrounding the well.

If the injected water has low TSS, but the injection rate is high, silt particles and clay colloids in receiving alluvial formations can be disturbed and clog the pores of the injection zone. Note that sites with alluvial formations are the most common to report clogging in the survey. Mechanical clogging problems also include air entrainment and formation particle jamming [21].

It should be noted that the pores of aquifers are typically too small to permit movement of the suspended particles for any distance beyond the borehole. Plugging caused by suspended solids can be mitigated by frequent re-development of the well during injection operation. Brown et al. [23] reported that well clogging issues have been managed successfully through the use of a regular back flushing programs. When the water flow is reversed, the particles are removed from the well screen, gravel, and/or borehole wall, along with the tightening of the gravel pack.

Re-development of the wells often entails discontinuing injection for a brief period, and reversing the well pump to remove solids accumulating around the wellbore. Periodic re-development should be expected but constant re-development hinders the goal of operations. For example, the City of Phoenix (AZ) had to backflush every few days, so the projects were finally abandoned [10] [11]. A number of the California systems have also reported clogging and two noted that re-development was required every 3 to 5 days to maintain well performance [24].

Chemical clogging is a geochemical issue. High conductivity water generally indicates the presence of chlorides and dissolved metals in the water. High TDS in itself is unlikely to create clogging problems, but it may contribute to chemcial clogging problems by bringing ions into the formation that can create various chemical reactions. When injected water with high cation concentrations replaces the more stable native water, geochemical equilibrium would need to be re-stablished through chemical reaction between the injected water and native fluid/aquifer matrix. In some cases, geochemical inequilibrium may result in the release of metals from the formation. Injection waters containing high sodium concentrations may also exacerbate clogging by hydrating and swelling of clays. Chemistry induced clogging is not unusual and may be treated with acidization of the well and re-development. For example, for the Wildwood, NJ system, the oldest ASR system in the US, acidization has been deployed every three months for each of its 4 ASR wells to combat clogging [25].

Microbiological fouling is generally interrelated with physical and chemical processes, and is particularly common with steel casings [13]. Microbiological fouling issues with ASR wells are similar to those encountered by water supply wells in that microorganisms can incrust or corrode the wells, and thus enhancing physical and chemical deterioration of the wells. Microbiological fouling encourages changes in the electrical potential and structure of the well material by creating anodes on metallic surfaces. Biofilms can form that eventually clog screens. The bacteria absorb nutrients and minerals such as iron, manganese, arsenic, nitrogen, and oxygen within the biofilm matrix, leading to the formation of tubercles and films that reduce capacity of the pumps and casings. The typical symptoms of microbiological fouling problems are [1]:

- Decrease in the water quality;

- Reduced specific capacity;

- Change in the amount of iron or manganese in the water supply;

- Increases in microbiological densities such as an observance of slimes or staining from the raw water.

Biological growth can be controlled by maintaining a chlorine residual in the injected water, but this can lead to disinfection by-products (DBPs) issues. 


\subsection{Metals Leaching/Water Quality}

A second problem observed among some of the ASR projects is water quality issues. Seventeen sites reported issues associated with water quality changes, particularly metals mobilization. Arsenic mobilization in limestone was reported in 11 Florida wells and two Wisconsin wells [4]-[6]. The metals leaching is most common with Florida and limestone, because virtually all the Florida wells use the same Floridan formation that contains pyrite in the limestone matrix. With respect to the Florida arsenic issues, Arthur et al. [4] reported that

“... examples of natural arsenic mobilization mechanisms and associated mineral phases include: 1) oxidation of sulfide minerals such as pyrite, which may contain trace elements as lattice substitutes, impurities or in solid solution (e.g., nickel, copper, cobalt, lead, arsenic, zinc and manganese), 2) desorption or dissolution of iron and manganese hydroxides (including grain or fracture coatings), 3) oxidation-reduction of organic material, which can mobilize organically complexed arsenic, and 4) biological transformations.”

The study suggests that

“... [f]rom the whole rock geochemistry data, many trace metals... showed high concentrations, when compared to global averages for limestones, especially arsenic in arsenian pyrite or organic materials” [4].

The results of their investigation indicated that geologists should assess chemical (including isotopic) variability within ground water and the aquifer system in light of the quality of the water to be injected. Of particular concern is the injection of oxygen-rich surface waters into an aquifer which can cause the release of trace metals into the injected water because of chemical in-equilibrium and reactions.

Brown et al. [23] reported that several ASR project sites in Oregon and Washington with naturally occurring radon in the recovered water. Eastern Municipal Water District in California had manganese issues when they started their program [26]. The ASR site located in Monmouth County, New Jersey had high iron in the recovered waters as a result of oxidation of minerals within the aquifer by oxygen in the recharge water [27]. As the iron concentrations in the recovered water increased considerably above the secondary drinking water standard of $0.3 \mathrm{mg} / \mathrm{L}$, the increases oxygen was suggested as a cause [27]. When the acidification treatment failed to correct the problems in Monmouth County, the iron concentration was reduced in the recovered water by elevating the $\mathrm{pH}$ of the recharge water [28]. The Foothill Municipal Water District ASR site in California reported elevated mercury levels that exceed historical levels in both the recharge water and the recovered native groundwater [27]. They used imported water sources for blending to bring the concentration down in the finished water to resolve the problem.

Geochemistry is a difficult and complex issue to work with among ASR wells. For successful ASR operations, the injection water must be compatible with the native groundwater and the aquifer material. Pyne [27] suggested $\mathrm{pH}$ differences between the recharge and groundwater can cause reactions that lead to clogging problems or the release of constituents (such as arsenic) from the aquifer matrix which may require removal upon recovery of the water. As noted in Table 6 and Table 7, surface waters were more likely to be associated with metals leaching problems (when compared with the use of groundwater, with the exception of Florida). It is suggested that this maybe because the dissolved oxygen content of surface waters is higher than native groundwater.

Chemical leaching issues can be solved using treatment techniques prior to injection and upon withdrawal. Overcoming geochemical problems may require modifications to the injected water-e.g., additional chemicals can be used to make the quality of injected water "match" more closely with that of the native groundwater.

\subsection{Disinfection Byproducts}

Potable and reclaimed water sources often contain chlorine that is used as a disinfectant. In the presence of organics, disinfection by-products (DBPs) such as haloacetic acids (HAAs) and trihalomethanes (THMs) would be generated. The presence of HAAs and THMs in the injection formation may be considered by regulatory agencies as introduced contaminants. The presence of DBPs (in the injection water) has led to stoppage of several projects in Washington and California. This issue was also raised in Florida and Colorado. Willows Water District in Denver, Colorado, Las Vegas, Nevada and Lancaster, California have all been studied in detail with regard to DBP issues [17] [29]-[31]. These studies and others demonstrated that microbial processes can attenuate HAA and THM during storage in the aquifer [17] [32]-[34] Pavelic et al. [35] reported that "three orders of magnitude difference in total THM attenuation rates occurred between the smallest half-lives at Charleston (less 
than 1 day) and the greatest half-lives at East Bay (120 to 550 days) and Las Vegas (greater than 120 days). There has been little evidence to suggest that the formation of DBPs continues in aquifers during ASR, apart from studies by Singer et al. [32] and Fram et al. [36] that were designed specifically to observe the kinetics involved with DBP formations. Other than those studies designed to deliberately create DBPs, only the Foothill Municipal Water District in California has observed an increase in THMs [37], which may likely be related to organic content in the injection horizon.

\subsection{Operational Issues}

Parsing through the data did indicate several trends:

- ASR sites with problems generally have a smaller number of injection wells. There were 2.4 wells per site with failure issues versus 4.4 at active sites. More successful sites tended to have more wells, for example, Grand Strand in South Carolina has 20 wells. This was not a surprise, but confirmed the expectations of the investigators.

- Sites with high injection or withdrawal rates tended to be less successful. The typical ASR wells injected or withdrew 1.5 MGD.

- The formation did not seem to matter much although the more common limestone and alluvial formations had issues with metal mobilization and clogging, respectively.

- The amount of water stored was lower for the inactive sites, which was not unexpected.

There are no big surprises in this data, but there may be an underlying anecdotal pattern that may worth investigating further. Newer projects were more likely to be stopped than older projects and thus the time period of inactive sites is generally shorter. The inactive southeast Florida wells that had recovery issues were all stopped within a three years of effort. Having evaluated numerous ASR projects and on the basis of discussion with project clients, the stoppage after a short time period may be related to client expectations on the ability to recover injected water (i.e., the willingness to inject a lot of water that might not be recovered and the willingness to spend money on a "risky" venture and anticipated treatment needs).

The inability to recover sufficient quantities of injected water was listed as a reason to abandon ASR efforts at 20 sites, primarily in Colorado and Florida. For recovery success, finding a confining layer immediately above the injection horizon is necessary so the buoyancy of the injected water does not cause the injected water to move away from the ASR well as shown to occur in two sites [38]. Another challenge associated with recovered water is the need to treat the water upon withdrawal, which is an issue with all the sites with arsenic problems. If the project client is unwilling to treat the recovered water, the project will not be a success. To deal with these issues, appropriate stratigraphy is required and a series of injection and recovery cycles must be undertaken to determine quality of the recovered water, the amount of water that might be successfully recovered and any needed treatment to meet compliance requirements. In order not to waste water, injection and recovery cycles usually start with small amount of water (e.g., under 10 million gallons) and then increase to 30 - 50 million gallons. To date, many of the systems have over 100 million gallons invested in injection cycles. Given most of the wells are designed to inject 1 MGD, 100 million gallons would take 100 days of injection. Recovery, however, may be more or less than the 1 MGD—one of the unknown and uncertain variables [2] [3] [36]. A 5 cycle test program might take 2 or 3 years on top of the permit and construction period. Such a program cost could cost $\$ 1$ - $\$ 1.5$ million. On the other hand, a client may expect that a well will be operational in a matter of a year or less, which is rarely attainable.

The use of ASR technology in brackish aquifer systems presents different challenges that are related to the mixing of the injected "fresh" water with the native brackish water. Where a brackish aquifer is used, the density difference between the treated freshwater and saline source would cause a "bubble" of freshwater to form. A large mixing area may also form as a result, depending on the formation, but with adequate quantities of injected water and the right aquifer properties, the bubble would remain fresh for a sufficiently long time period to be available for water supply augmentation. The quantity of water to be stored can be as extensive as the localized aquifer system allows, and can be continuously pumped into the aquifer system until needed. However, the initially mixed water may not be recoverable unless treatment is deployed to remove impurities such as elevated levels of chlorides (salinity). In many cases, the transition zone between native an fresh water may be over 500 million gallons for a single well [2] [36], after which the ASR system may derive 70 percent recovery of the water injected, depending on aquifer characteristics. 
An ongoing current issue in Texas, among others is tied to ultimate management of the waters recharged. While no utility has noted that this as a barrier, several states are in the process of, or have had to revise, waterrights laws identifying who owns the water recharged in an ASR system. Those rules are ongoing and state specific. If a utility cannot recover the water it injects because of water-rights issues, the incentive to pursue ASR is lost.

\section{Conclusions}

This paper results from a nationwide analysis of ASR systems through a data gathering effort as of July 1, 2013. Unlike the previous efforts, every utility that was identified as having investigated ASR was contacted, whether they proceeded with the development of ASR projects or not, and whether the system was still active or not. Nine months of data collection, literature reviews, online searches, phone calls, emails, file searches and permit collection yielded information on operations of the ASR systems, stratigraphy, ASR challenges and information were gathered and compiled for every system we knew of in the U.S. Copies of reports and rules have been posted at www.h2o-pe.com. One of the issues that arise from the inventory of the 204 systems in the U.S. is that $26 \%$ percent of the ASR projects have been functionally abandoned.

This paper focuses on the causes of failures associated with ASR projects and provides solutions to avoid some of the common pitfalls. While the wells at over 50 sites were technically abandoned, only one (Broward County) was reported as permanently plugged and abandoned, and three systems in California were converted to potable supply wells, which was not surprising because many of the California ASR wells started as supply wells. Geochemistry, clogging and recovery are the most common issues associated with failed efforts. Both physical and chemical properties of the injected water may change after injection as a function of chemical reactions between injected water and the native groundwater/aquifer matrix. The stored water has the potential to change as a result of increased aquifer pressure, temperature (increases or decreases), and the potential for micro-organisms activities. The changes in water and the formation are directly related to recovery potential.

The combined challenges of meeting requirements for drinking water storage, groundwater injection, water quality and disinfection byproducts rose in several states. The need to establish ownership of the stored water in several states has created additional challenges that must be addressed by the regulatory agencies within those states where ASR is used and water rights are at issue.

\section{References}

[1] American Water Works Association (2014) M21 Groundwater. AWWA, Denver.

[2] Bloetscher, F., Muniz, A. and Witt, G.M. (2005) Groundwater Injection: Modeling, Risks, and Regulations. McGrawHill, New York.

[3] Bloetscher, F., Sham, C.H., Ratick, S. and Danko III, J.J. (2014) Status of Aquifer Storage and Recovery in the United States-2013. JAWWA.

[4] Arthur, J.D., Cowart, J.B. and Dabous, A. (2001) Florida Aquifer Storage and Recovery Geochemical Study: Year Three Progress Report. Florida Geological Survey Open File Report 83, 48 p. http://water.usgs.gov/ogw/pubs/ofr0289/jda_mobilization.htm

[5] Reese, R. (2002) Inventory and Review of Aquifer Storage and Recovery in Southern Florida. U.S. Geological Survey Water-Resources Investigations Report 02-4036, USGS, Tallahassee.

[6] Austin, B.P. (2013) Email to F. Bloetscher Dated 4/10/13 Wisconsin ASR Wells.

[7] United States Geological Survey (1995) Highline Well Field Aquifer Storage and Recovery Project Seattle, Washington. Final Report, U.S. Department of the Interior Bureau of Reclamation Snake River Area Office-West and Pacific Northwest Regional Office Boise, Idaho.

[8] Pyne, R.D.G. (2013) Email to F. Bloetscher Dated 6/8/13 Seattle Highline ASR Wells.

[9] Coates, M., Lehman, P., Varn, C. and Manson, D. (2013) New Path to Permitting Aquifer Storage and Recovery Systems in Florida. Florida Water Resources Journal, 65, 10.

[10] Gin, G. (2013) Email to F. Bloetscher Dated 7/1/13 Phoenix ASR Wells.

[11] Gin, G. (2013) Email to F. Bloetscher Dated 5/6/13 Phoenix ASR Wells.

[12] Walker, D. (2013) Email to F. Bloetscher Dated 7/25/13 Phoenix ASR Wells.

[13] Bloetscher, F. and Witt, G.M. (1997) Aquifer Storage and Recovery as a Means of Resource Management: A Summary. 
AWWA Annual Conference and Exposition Proceedings, Atlanta, June 1997.

[14] Boomgaard, C. (2013) Email to F. Bloetscher Dated 3/21/13 Various CO ASR Wells.

[15] Bloetscher, F. and Muniz, A. (2010) Summary of ASR Technology. Sustainable Water Sources Conference Proceedings, Albuquerque, April 2010.

[16] Pyne, R.D.G. (2013) Email to F. Bloetscher Dated 9/3/13 N. Georgia ASR Well.

[17] Pyne, R.D.G. (2013) Email to F. Bloetscher Dated 9/27/13 N. Georgia ASR Well.

[18] Thomas, J.M., McKay, W.A., Cole, E., Landmeyer, J.E. and Bradley, P.M. (2000) The Fate of Haloacetic Acids and Trihalomethanes in an Aquifer Storage and Recovery Program, Las Vegas, Nevada. Groundwater, 38, 605-614.

[19] USEPA (1999) Class V Underground Injection Control Study: Volume 21, Aquifer Recharge and Aquifer Storage and Recovery Wells. Office of Ground Water and Drinking Water, EPA/816-R-99-014u, Washington DC.

[20] Pyne, R.D.G., Singer, P.C. and Miller, C.T. (1996) Aquifer Storage and Recovery of Treated Drinking Water. American Water Works Association, Publication No. 90689, AWWA, Denver.

[21] Pitt, W.A.J. and Magenheimer, S. (1997) ASR Technology: Avoidance and Solutions to Aquifer Clogging Problems. Proceedings of AWRA Symposium, Conjunctive Use of Water Resources: Aquifer Storage and Recovery, Long Beach, 19-23 October 1997, 251-260.

[22] Pyne, R.D.G. (1995) Groundwater Recharge and Wells: A Guide to Aquifer Storage Recovery. Lewis Publishers, Boca Raton.

[23] Brown, C.J., Hatfield, K. and Newman, M. (2006) Lessons Learned from a Review of 50 ASR Projects from the United States, England, Australia, India, and Africa. University of North Florida, Jacksonville.

[24] Rydman, D. (2012) Lessons Learned from and Aquifer Storage and Recovery Program. Journal of the American Water Works Association, 104, 52-57.

[25] Pyne, R.D.G. (2007) Overview of Aquifer Storage Recovery in the USA and the World. Conference on Aquifer Storage Recovery and Artificial Recharge in Oregon, Overcoming Technical, Regulatory and Social Challenges, 7 November 2007. http://water.oregonstate.edu/asr/presentations/Pyne.pdf

[26] Wutting, M. (2013) Email to F. Bloetscher Dated 8/1/13 California Wells, and Subsequent Phone Call.

[27] Pyne, R.D.G., SJRWMD (2004) Aquifer Storage and Recovery (ASR) Issues and Concepts. A Position Paper Prepared by the St. Johns River Water Management District, SJRWMD, Jacksonville.

[28] Lucas, M. and McGill, K. (1997) Aquifer Treatment Methods for Preventing Iron Concentrations in the Recovered Water from Aquifer Storage and Recovery Wells Installed in Clayey Sand Aquifers of the Atlantic Coastal Plain. Proceedings of AWRA Symposium, Conjunctive Use of Water Resources: Aquifer Storage and Recovery, Long Beach, 19-23 October 1997, 449.

[29] Mille, C.J., Wilson, L.G., Amy, G.L. and Brothers, K. (1993) Fate of Organochlorine Compounds during Aquifer Storage and Recovery: The Las Vegas Experience. Groundwater, 31, 410-416. http://dx.doi.org/10.1111/j.1745-6584.1993.tb01843.x

[30] Katzer, T. and Brothers, K. (1989) Artificial Recharge in Las Vegas Valley, Clark County, Nevada. Groundwater, 27, 50-56. http://dx.doi.org/10.1111/j.1745-6584.1989.tb00007.x

[31] Baqai, H.A. (2002) Effects of Recharge of Chlorinated State Water Project Waters to Groundwaters in Lancaster Area of California. Water Engineering and Management, 149, 10-13.

[32] Singer, P.C., Pyne, R.D.G., Mallikarjun, A.V.S., Miller, C.T. and Mojonnier, C. (1993) Examining the Impact of Aquifer Storage and Recovery on DBPs. Journal of the American Water Works Association, 85, 85-94.

[33] Bouwer, E.J. and Wright, J.P. (1987) Transformation of Trace Halogenated Aliphatics in Anoxic Biofilm Columns. Journal of Contaminant Hydrology, 2, 155-169. http://dx.doi.org/10.1016/0169-7722(88)90005-8

[34] Landmeyer, J.E., Bradley, P.M. and Thomas, J.M. (2000) Biodegradation of Disinfection Byproducts as a Removal Process during Aquifer Storage and Recovery. JAWRA, 36, 861-867.

[35] Pavelic, P., Dillon, P. and Nicholson, B. (2007) Formation and Attenuation of DBPs in Groundwater at ASR Sites. ISMAR6 Proceedings, Phoenix, 8 October-2 November 2007, 613.

[36] Fram, M.S., Bergamaschi, B.A., Goodwin, K.D., Fujii, R. and Clark, J.F. (2003) Processes Affecting the Trihalomethane Concentrations Associated with the Third Injection, Storage and Recovery Test at Lancaster, Antelope Valley, California, March 1998 through April, 1999. United States Geological Survey Water Resources Investigations Report 03-4062.

[37] Palmer, R., Wendell, D., Hutchinson, A. and Foreman, T. (1997) Optimization of ASR Operations in a Nitrate and PCE Contaminated Aquifer. Proceedings of AWRA Symposium, Conjunctive Use of Water Resources: Aquifer Storage and Recovery, Long Beach, 19-23 October 1997, 3-11. 
[38] Bloetscher, F., Muniz, A. and Gonzalez, A.M. (2003) Summary of AWWA Survey of ASR Practices in the United States. Florida Water Resource Conference Proceedings, Tampa, Florida, FWRC, St. Cloud.

[39] Hosmer, D.W. and Lemeshow, S. (1980) Goodness-of-Fit Test for the Multiple Logistic Regression Model. Communications in Statistics, 9, 1043-1069. http://dx.doi.org/10.1080/03610928008827941

[40] Cox, D.R. and Snell, E.J. (1989) Analysis of Binary Data. 2nd Edition, Chapman \& Hall, London.

[41] Nagelkerke, N.J.D. (1991) A Note on a General Definition of the Coefficient of Determination. Biometrika, 78, 691692. http://dx.doi.org/10.1093/biomet/78.3.691 
Scientific Research Publishing (SCIRP) is one of the largest Open Access journal publishers. It is currently publishing more than 200 open access, online, peer-reviewed journals covering a wide range of academic disciplines. SCIRP serves the worldwide academic communities and contributes to the progress and application of science with its publication.

Other selected journals from SCIRP are listed as below. Submit your manuscript to us via either submit@scirp.org or Online Submission Portal.
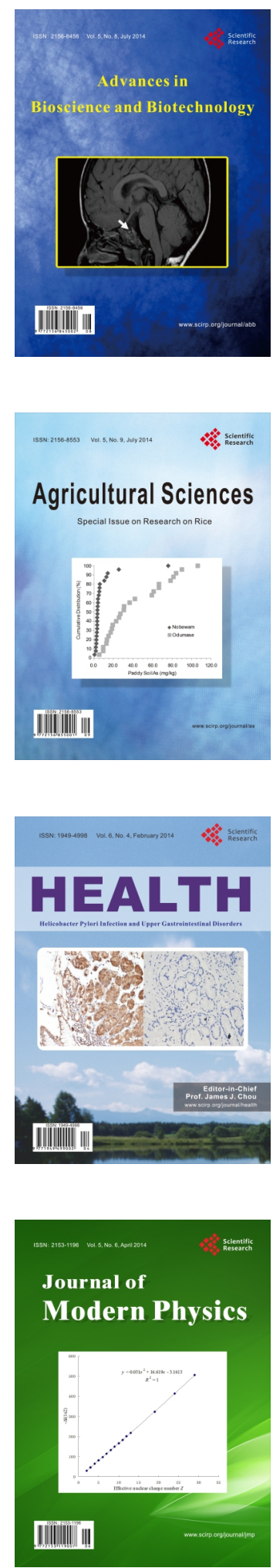
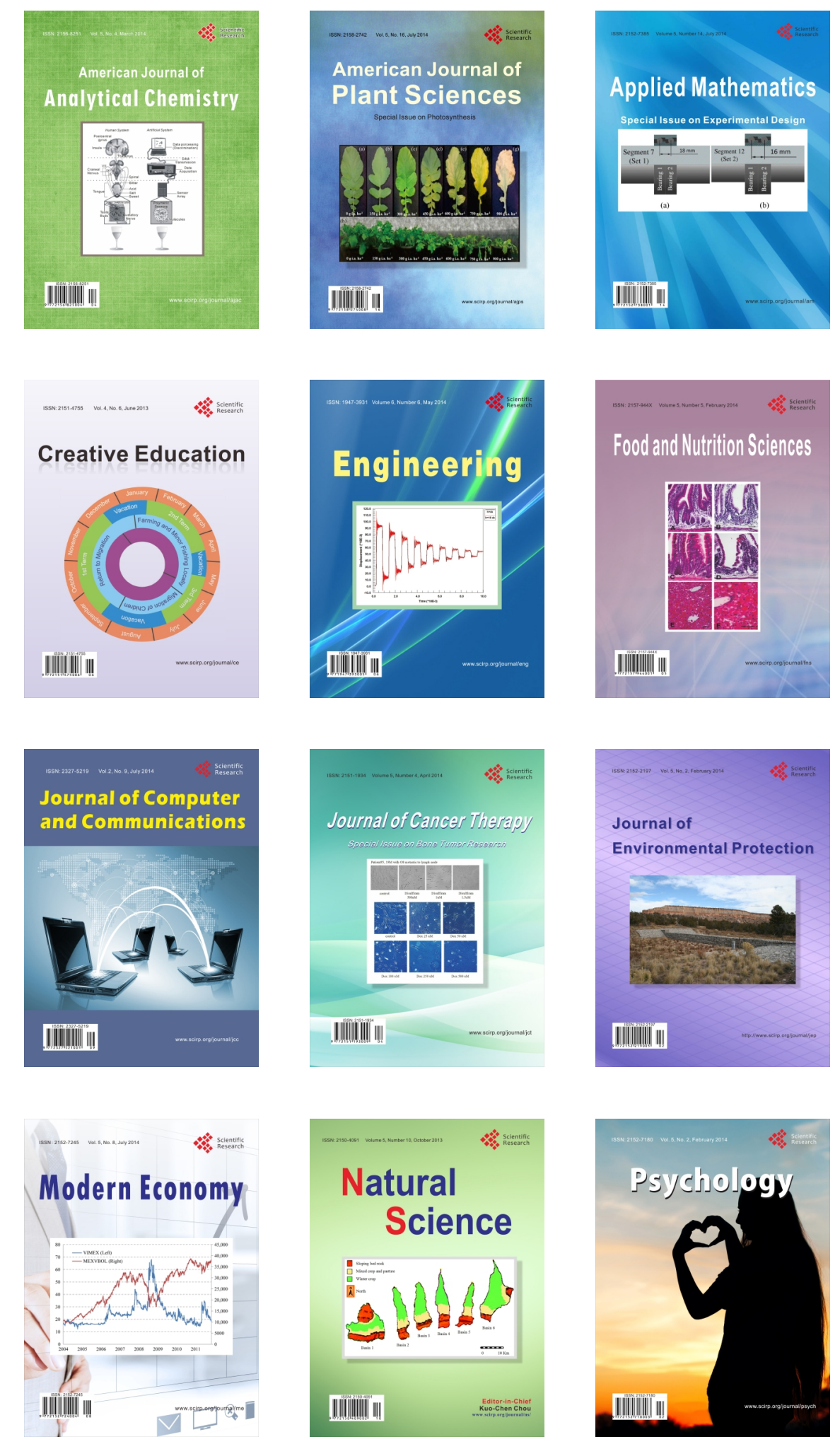